\title{
Nonlinear Stability of Discrete Shocks for Systems of Conservation Laws
}

\author{
JiAN-Guo LIU \& Zhouping XIN
}

Communicated by C. DAFERMOS

\begin{abstract}
In this paper we study the asymptotic nonlinear stability of discrete shocks for the Lax-Friedrichs scheme for approximating general $m \times m$ systems of nonlinear hyperbolic conservation laws. It is shown that weak single discrete shocks for such a scheme are nonlinearly stable in the $L^{p}$-norm for all $p \geqq 1$, provided that the sums of the initial perturbations equal zero. These results should shed light on the convergence of the numerical solution constructed by the Lax-Friedrichs scheme for the single-shock solution of system of hyperbolic conservation laws. If the Riemann solution corresponding to the given far-field states is a superposition of $m$ single shocks from each characteristic family, we show that the corresponding multiple discrete shocks are nonlinearly stable in $L^{p}(p \geqq 2)$. These results are proved by using both a weighted estimate and a characteristic energy method based on the internal structures of the discrete shocks and the essential monotonicity of the Lax-Friedrichs scheme.
\end{abstract}

\section{Introduction}

We investigate the asymptotic stability of the numerical approximation of the following Riemann problem for general systems of nonlinear conservation laws

$$
u_{t}+f(u)_{x}=0, \quad u(x, 0)=u_{0}(x)= \begin{cases}u_{-}, & x<0 \\ u_{+}, & x>0\end{cases}
$$

where $u=u(x, t) \in \mathbf{R}^{m}, f$ is a smooth nonlinear mapping from $\mathbf{R}^{m}$ to $\mathbf{R}^{m}$, and $u_{ \pm}$are two constant vectors in $\mathbf{R}^{m}$. We assume that the system (1.1) is strictly hyperbolic in the sense that at each state $u \in \mathbf{R}^{m}$ the Jacobian $\nabla f(u)$ has $m$ real and distinct eigenvalues

$$
\lambda_{1}(u)<\lambda_{2}(u)<\ldots<\lambda_{m}(u)
$$


with corresponding left and right eigenvectors $l_{\mu}(u)$ and $r_{\mu}(u)$, respectively, and that each characteristic field is either genuinely nonlinear or linearly degenerate in the sense of LAX [14], i.e., for $\mu=1, \ldots, m$, the eigenvector $r_{\mu}$ satisfies $\nabla \lambda_{\mu} \cdot r_{\mu} \equiv 1$ or $\nabla \lambda_{\mu} \cdot r_{\mu} \equiv 0$. We normalize the eigenvectors so that $l_{\mu}(u) r_{k}(u)=\delta_{\mu k}$ and denote the $m \times m$ matrices $L(u), R(u)$ and $\Lambda(u)$ by

$$
\begin{gathered}
L(u)=\left(l_{1}(u)^{\top}, \ldots, l_{m}(u)^{\top}\right)^{\top}, \quad R(u)=\left(r_{1}(u), \ldots, r_{m}(u)\right), \\
\Lambda(u)=\operatorname{diag}\left(\lambda_{1}(u), \ldots, \lambda_{m}(u)\right) .
\end{gathered}
$$

LAX [14] showed that the Riemann problem (1.1) has a solution consisting of at most $(m+1)$ constant states separated by shock waves, centered rarefaction waves, and contact discontinuities, provided that the shock strength $\left|u_{+}-u_{-}\right|$ is small.

We focus mainly on two special cases, the single shock and the multipleshock. In the first case, the Riemann problem (1.1) has a $k$-shock wave solution

$$
u(x, t)= \begin{cases}u_{-}, & x<s t, \\ u_{+}, & x>s t,\end{cases}
$$

corresponding to the $k$-th genuinely nonlinear field. Here the constant states $u_{ \pm}$and shock speed $s$ satisfy the Rankine-Hugoniot condition

$$
f\left(u_{-}\right)-f\left(u_{+}\right)=s\left(u_{-}-u_{+}\right),
$$

and the Lax entropy condition

$$
\lambda_{k}\left(u_{+}\right)<s<\lambda_{k}\left(u_{-}\right) .
$$

In the second case, the Riemann problem (1.1) has a multiple shock solution

$$
u(x, t)=\left\{\begin{array}{cc}
\bar{u}_{0}, & x<s_{1} t, \\
\bar{u}_{1}, & s_{1} t<x<s_{2} t, \\
\vdots & \vdots \\
\bar{u}_{m-1}, & s_{m-1} t<x<s_{m} t, \\
\bar{u}_{m}, & s_{m} t<x .
\end{array}\right.
$$

Here the constant states $\bar{u}_{0}=u_{-}, \bar{u}_{1}, \ldots, \bar{u}_{m-1}, \bar{u}_{m}=u_{+}$and shock speeds $s_{1}, \ldots, s_{m}$ satisfy the Rankine-Hugoniot conditions

$$
f\left(\bar{u}_{\mu-1}\right)-f\left(\bar{u}_{\mu}\right)=s_{\mu}\left(\bar{u}_{\mu-1}-\bar{u}_{\mu}\right), \quad \mu=1, \ldots, m,
$$

and the Lax entropy conditions

$$
\lambda_{\mu}\left(\bar{u}_{\mu}\right)<s_{\mu}<\lambda_{\mu}\left(\bar{u}_{\mu-1}\right), \quad \mu=1, \ldots, m .
$$

The multiple shock is generic in the sense that it is preserved under small perturbations of the left and right constant states $u_{-}$and $u_{+}$.

We approximate (1.1) with the difference schemes (consistent and conservative) of the form

$$
u_{j}^{n+1}=G\left(u_{j-r}^{n}, \ldots, u_{j}^{n}, \ldots, u_{j+t}^{n}\right)
$$


where $G\left(u_{-r}, \ldots, u_{t}\right): \mathbf{R}^{(r+t+1) m} \rightarrow \mathbf{R}^{m}$ is a smooth function. In particular, we take the Lax-Friedrichs scheme as the approximation scheme [13]

$$
u_{j}^{n+1}-u_{j}^{n}+\frac{\lambda}{2}\left(f\left(u_{j+1}^{n}\right)-f\left(u_{j-1}^{n}\right)\right)=\frac{\nu}{2}\left(u_{j+1}^{n}-2 u_{j}^{n}+u_{j-1}^{n}\right)
$$

where $u_{j}^{n}$ is a approximation of $u\left(x_{j}, t_{n}\right), x_{j}=j \Delta x$ and $t_{n}=n \Delta t$, and $\Delta x$ and $\Delta t$ are the spatial and temporal grid sizes; $v$ is a constant satisfying $0<v<1$, and the temporal-spatial grid ratio $\lambda=\Delta t / \Delta x$ satisfies a Courant-FriedrichsLewy condition

$$
\lambda \sup _{\mu}\left|\lambda_{\mu}(u)\right| \leqq \nu .
$$

A solution $\phi_{j}^{n}$ of

$$
\begin{gathered}
\phi_{j}^{n+1}-\phi_{j}^{n}+\frac{\lambda}{2}\left(f\left(\phi_{j+1}^{n}\right)-f\left(\phi_{j-1}^{n}\right)\right)=\frac{v}{2}\left(\phi_{j+1}^{n}-2 \phi_{j}^{n}+\phi_{j-1}^{n}\right), \\
\phi_{j}^{n q}=\phi_{j-n p}^{0}, \\
\phi_{j} \rightarrow u_{ \pm} \quad \text { as } j \rightarrow \pm \infty
\end{gathered}
$$

is called a traveling-wave solution. It is referred to as a single discrete shock. Its existence has been proved by MAJDA \& RALSTON [18] provided that $\left(u_{-}, u_{+}\right)$satisfies condition (1.2). Here, $\lambda s=p / q$ for some relatively prime integers $p$ and $q$.

The first main theorem of this paper is the following; it gives the asymptotic $L^{2}$-stability and $L^{1}$-stability of single discrete shock waves for the LaxFriedrichs scheme.

Theorem 1.1. Suppose that (1.1) is a strictly hyperbolic system and that the $k$ characteristic field is genuinely nonlinear. Let $\phi_{j}^{n}$ be the single discrete shock profile (1.6) in the $k$-field connecting $u_{+}$to $u_{-}$. Assume that

$$
\begin{gathered}
\sum_{j=-\infty}^{\infty}\left(u_{j}^{0}-\phi_{j}^{0}\right)=0, \\
\varepsilon=\left|u_{+}-u_{-}\right| \leqq c_{1}, \\
\sum_{j=-\infty}^{\infty}\left(1+j^{2}\right)\left|u_{j}^{0}-\phi_{j}^{0}\right|^{2} \leqq c_{2}
\end{gathered}
$$

for some (suitably small) positive constants $c_{1}$ and $c_{2}$. Then

(1) There exists a unique global solution $u_{j}^{n}$ to the Lax-Friedrichs scheme (1.5) with initial data $u_{j}^{0}$, which satisfies

(2) If

$$
\lim _{n \rightarrow \infty} \sum_{j=-\infty}^{\infty}\left|u_{j}^{n}-\phi_{j}^{n}\right|^{2}=0 .
$$

$$
\sum_{j=-\infty}^{\infty}\left(1+j^{2}\right)^{3 / 2}\left|u_{j}^{0}-\phi_{j}^{0}\right|^{2}<\infty,
$$


then

$$
\sup _{0 \leqq n<\infty} \sum_{j=-\infty}^{\infty}\left|u_{j}^{n}-\phi_{j}^{n}\right|<\infty .
$$

As an immediate consequence of Theorem 1.1, we have the following corollary.

Corollary 1.1. Under the hypothesis of Theorem 1.1, there is $L^{p}$ asymptotic stability for $p>1$ :

$$
\lim _{n \rightarrow \infty} \sum_{j=-\infty}^{\infty}\left|u_{j}^{n}-\phi_{j}^{n}\right|^{p}=0 .
$$

Remark 1.1. One would expect that the stability estimate (1.9) in Theorem 1.1 implies the following error estimate for the Lax-Friedrichs scheme (1.5a) approximating systems of conservation laws (1.1) with single-shock solution $u(x, t)$, of the form $(1.2 \mathrm{a})$ :

$$
\left\|u(\cdot, t)-u_{h}(\cdot, t)\right\|_{L^{1}} \leqq C h
$$

where $u_{h}(x, t)$ is the approximate solution and $C$ is a positive constant independent of the grid size $h$. The error estimate in (1.11) should be optimal. It has been achieved by JENNINGS in the scalar case [10]. It remains to combine some initial-layer estimates with (1.9) to obtain (1.11). This is left for the future.

Remark 1.2. In the original Lax-Friedrichs scheme [13], $v=1$. However, we do not expect asymptotic stability of the discrete shock profiles in this case. In fact, we can easily verify that stationary discrete shock profiles of the LaxFriedrichs scheme for scalar equations are not asymptotically stable [16]. We note that the theorem of JeNNINGs [10] for scalar equations also excludes the case $v=1$.

Next, we define the multiple discrete shock corresponding to the multiple shock solution (1.3) to be the superposition of $m$ single discrete shocks:

$$
\phi_{j}^{n}=\sum_{\mu=1}^{m} \phi_{\mu, j+\delta_{\mu}}^{n}-\sum_{\mu=1}^{m-1} \bar{u}_{\mu},
$$

where $\phi_{\mu, j}^{n}$ is the discrete shock connecting the constant states $\vec{u}_{\mu-1}$ and $\bar{u}_{\mu}$, and the $\delta_{\mu}$ are integers determined by initial data.

The second main theorem of this paper is the following asymptotic $L^{2}$-stability of the multiple discrete shock waves for the Lax-Friedrichs scheme.

Theorem 1.2. Suppose that system (1.1) is strictly hyperbolic and genuinely nonlinear. Let $\phi_{j}^{n}$ be the discrete multiple shock profile (1.12), and let integers $\delta_{\mu}$ be chosen such that

$$
\sum_{j=-\infty}^{\infty}\left(u_{j}^{0}-\sum_{\mu=1}^{m} \phi_{\mu, j+\delta_{\mu}}^{0}+\sum_{\mu=1}^{m-1} \bar{u}_{\mu}\right)=0 .
$$


Then there exist (suitably small) positive constants $c_{1}$ and $c_{2}$ such that if

$$
\begin{gathered}
\varepsilon=\left|u_{+}-u_{-}\right| \leqq c_{1}, \\
\sum_{j=-\infty}^{\infty}\left(1+j^{2}\right)\left|u_{j}^{0}-\sum_{\mu=1}^{m} \phi_{\mu, j+\delta_{\mu}}^{0}+\sum_{\mu=1}^{m-1} \bar{u}_{\mu}\right|^{2} \leqq c_{2},
\end{gathered}
$$

then there exists a unique global solution, $u_{j}^{n}$, to the Lax-Friedrichs scheme (1.5). Furthermore,

$$
\lim _{n \rightarrow \infty} \sum_{j=-\infty}^{\infty}\left|u_{j}^{n}-\phi_{j}^{n}\right|^{2}=0 .
$$

Our stability analysis is strongly motivated by the nonlinear stability of viscous shock waves for systems of conservation laws with viscosity of the form

$$
u_{t}+f(u)_{x}=v u_{x x}, \quad v>0 .
$$

There have been extensive studies of this question in the last three decades $[5,6,11,15,22,29]$. Recently, important progress has been made by GooDman [5], Kawashima \& Matsumura [11], Liu [15], and Szepessy \& XIN [27] in the study of asymptotic stability of viscous shock profiles for a large class of conservation laws with viscosity. These authors showed that a weak viscous shock profile is nonlinearly stable in the $L_{2}$-norm in the sense that a small initial disturbance, under suitable restrictions, dies out as time tends to infinity. In the scalar case, Jennings [10], Osher \& RaLston [22] proved $L^{1}$-stability for discrete shocks and viscous shocks, respectively.

The study of the existence and stability of discrete shocks is important in understanding the convergence behavior of numerical shock computations. In very interesting work [10], JENNINGS showed the stability of discrete shock profiles for general first-order monotone schemes for scalar conservation laws. The existence of discrete shock waves for finite-difference methods accurate to first order for systems of conservation laws was established by MAJDA \& RALSTON [18] using the center manifold theorem; see also [19]. ENGQUIST \& OSHER proved the stability of their first-order monotone scheme for the scalar case [4]. SMYRLIS [25] proved the stability of a scalar stationary discrete shock wave for the Lax-Wendroff scheme. SzEPESSY [26] studied the existence and $L^{2}$-stability of stationary discrete shocks for an implicit first-order, streamlinediffusion, finite-element method for systems of conservation laws. In contrast to [26], we deal here with explicit schemes and non-stationary shocks. We also treat the case of multiple shocks and $L^{1}$-stability. Although we have proved similar results for stationary discrete shocks for the Lax-Friedrichs scheme by a different (and simpler) method [16], which is of intrinsic interest, we cannot apply this method in [16] here due to the complicated structure of non-stationary discrete shocks. In the case that the given far field is a constant state, CHERN [1] proved the stability of the Lax-Friedrichs scheme using diffusion waves.

As is well known, the $L^{1}$-norm is the natural norm, both mathematically and physically, to measure the stability of shock waves. As far as we know, 
our $L^{1}$-stability result in Theorem 1.1 is the first for shock waves for systems of conservation laws. In the scalar case, JENNINGs obtained the same result [10].

The Lax-Friedrichs scheme has played a very important role, both in theoretical and numerical computations and in the development of the theory of hyperbolic systems of conservation laws. The scheme first appeared in LAX [13] and was used by OLEINIK [21] to prove the existence and uniqueness of the admissible solution for scalar conservation laws. DIPERNA used the LaxFriedrichs scheme to prove the existence of large-amplitude weak solutions for some $2 \times 2$ systems [3]. Nishida \& SMOLLER [20] also studied isentropic gas flows with a modified Lax-Friedrichs scheme.

For scalar conservation laws, the Lax-Friedrichs scheme belongs to the class of monotone schemes which are well understood; see [12, 2, 24], e.g. It has been shown by KUZNECOV [12] (see also SANDERS [24]) that the best rate of convergence in the $L^{1}$-norm for such a scheme with general BV initial data is $h^{1 / 2}$. Harabetian [8] studied the large-time behavior of rarefaction waves for some monotone schemes; see also [28].

In a forthcoming paper [17], we employ ideas developed in this paper to prove the convergence of approximations constructed by the Lax-Friedrichs scheme to piecewise smooth solutions for systems of conservation laws. GoonMAN \& XIN [7] have proved convergence of viscous solutions for systems of conservation laws to piecewise smooth inviscid flows.

The outline of this paper is as follows. In Section 2, we study some important properties of the discrete shock profiles constructed by MAJDA \& RALSTON. These properties, such as the compressibility of admissible shocks and their asymptotic behavior, play crucial roles in the stability analysis. The key step in establishing these properties is that, in the construction of center manifolds for the enlarged system, because the shock is non-stationary, we need careful analysis to show that waves in the transversal direction are of higher order than the principal wave. This fact, together with the entropy condition, essentially yields the desired properties.

We treat the $L^{2}$-stability of single shocks in Section 3 . As in $[5,15,27,29]$, we use an energy method. Because the scheme is explicit and the shock is nonstationary, a simple weighted energy estimate is not available here, and our analysis is technically more involved than its continuous counterpart and the analyses in $[16,26]$.

In Section 4 , we investigate the $L^{1}$-stability of single discrete shocks. In contrast to the scalar case, due to the coupling of waves from different characteristic families, even the linear stability analysis in the $L^{1}$-norm is very difficult. We overcome this difficulty by carefully choosing weights so that the propagation of the perturbations in the principal direction dominates the disturbance in the transversal wave directions. The perturbation in the principal direction can be estimated by using the essential monotonicity of the scheme in the principal direction. This, together with the $L^{2}$-nonlinear stability analysis, yields the desired result: Theorem 1.1.

The stability of multiple discrete shocks is treated in Section 5. Since the system is strictly hyperbolic, so that waves from different characteristic families propagate with distinct speeds, waves produced by interactions of waves of dif- 
ferent families are controllable both in space and time. This enables us to do a characteristic decomposition, so that each wave, on its dominant region, can be estimated essentially as in the single-shock case, while on the complement of its dominant region, it can be analyzed by a modified version of vertical estimates.

\section{Properties of Discrete Shock Profiles}

We are concerned with the finite difference schemes (consistent and conservative)

$$
u_{j}^{n+1}=G\left(u_{j-r}^{n}, \ldots, u_{j}^{n}, \ldots, u_{j+t}^{n}\right),
$$

approximating (1.1). We assume that $(2.1 \mathrm{a})$ is a first-order scheme and satisfies

$$
\kappa(u) \equiv l_{k}(u) \sum_{i=-r}^{t} i^{2} C_{i}(u) r_{k}(u)-\left|\lambda \lambda_{k}(u)\right|^{2}>0
$$

where $C_{i}(u)=\left.\partial_{u_{i}} G\left(u_{-r}, \ldots, u_{t}\right)\right|_{u_{-r}=u_{1}, \ldots, u_{t}=u}$. The discrete shock is a traveling-wave solution of (2.1) satisfying

$$
\phi_{j-p}=G^{q}\left(\phi_{j-r q}, \ldots, \phi_{j+t q}\right), \quad \phi_{j} \rightarrow u_{ \pm} \text {as } j \rightarrow \pm \infty .
$$

We recall that $p$ and $q$ are integers that satisfy $\lambda s=p / q$. The existence of discrete shocks for (2.1) has been proved by MAJDA \& RALSTON [18] using a center manifold theorem. In this section, we refine the center manifold construction, show the strict monotonicity of the principal eigenvalues at the discrete shock profile, and discuss some asymptotic properties of the profiles.

Theorem 2.1 (MAJDA \& RALSTON [18]). Let $u_{-}$and $u_{+}$be the left and right states of a k-shock (1.2) with shock speed s. Suppose that the shock strength $\varepsilon=\left|u_{+}-u_{-}\right|$is small and that $s \Delta t \mid \Delta x=p / q, p, q \in \mathbb{Z}$. Then there is a family. of discrete shock profiles $\phi_{j}$ satisfying (2.2).

MAJDA \& RALSTON proved this theorem by applying a center-manifold theorem (Proposition 2.1 below) to the marching map

given by

$$
T: \mathbf{R}^{(r+t) m q} \rightarrow \mathbf{R}^{(r+t) m q}
$$

in which

$$
T\left(\begin{array}{c}
u_{1} \\
\vdots \\
u_{(r+t) q}
\end{array}\right)=\left(\begin{array}{c}
u_{2} \\
\vdots \\
u_{(r+t) q} \\
H\left(u_{1}, \ldots, u_{(r+t) q}\right.
\end{array}\right),
$$

$$
\phi_{t q}=H\left(\phi_{j-r q}, \ldots, \phi_{j+t q-1}\right)
$$

is a traveling-wave profile which is equivalent to the standard form

$$
\phi_{j-p}=G^{q}\left(\phi_{j-r q}, \ldots, \phi_{j+t q}\right) \text {. }
$$

One can verify that the marching map $T$ belongs to the class of operators defined below (Lemma 3.1 in [18]). 
Let $T: \mathbf{R}^{N} \rightarrow \mathbf{R}^{N}$ be a smooth map and $x_{0} \in \mathbf{R}^{N}$ be a fixed point of $T$ so that the following conditions are satisfied.

(i) There are neighborhoods $\mathscr{U}$ and $\mathscr{U}^{\prime}$ of $x_{0}$ in $\mathbf{R}^{N}$ such that $T$ maps $\mathscr{U}$ diffeomorphically onto $\mathscr{U}^{\prime}$.

(ii) $T$ fixes a smooth $m$-dimensional manifold $\Omega$ containing $x_{0}$.

(iii) The algebraic eigenspace of $d T\left(x_{0}\right)$ associated with the eigenvalue 1 is the span of the tangent space of $\Omega, \mathscr{F}_{x_{0}}(\Omega)$, and a vector $\chi$ satisfying

$$
d T\left(x_{0}\right) \chi=\chi+r
$$

where $r \in \mathscr{T}_{x_{0}}(\Omega)$ and $r \neq 0$.

(iv) $d T\left(x_{0}\right)$ has no eigenvalues in the set $\{z:|z|=1, z \neq 1\}$.

(v) Hypotheses (ii) and (iii) imply that, given $\delta^{\prime}>0$, there is a $\delta>0$ such that, when $x \in \Omega$ and $\left|x-x_{0}\right|<\delta$, the eigenvalues of $d T(x)$ lying in $|z-1|<\delta^{\prime}$ are 1 with multiplicity $m$ and $\beta(x)$ with multiplicity 1 where $\beta(x) \in C^{\infty}(\Omega)$.

We assume that

$$
r \cdot \nabla \beta\left(x_{0}\right)<0 .
$$

We summarize the center manifold constructions for such an operator in [18] in the following proposition.

Proposition 2.1 [18]. Assume that the map $T$ satisfies conditions (i) $-(\mathrm{v})$. Then there is an $\varepsilon>0$ such that, if $x_{-} \in \Omega, \beta\left(x_{-}\right)>1$ and $\left|x_{-}-x_{0}\right|<\varepsilon$, then

(1) there is a $C^{1}$ segment $\gamma_{x_{-}}$beginning at $x_{-}$such that $T^{-1} \gamma_{x_{-}} \subset \gamma_{x_{-}}$and $\bigcap_{j} T^{-j} \gamma_{x_{\ldots-}}=\left\{x_{-}\right\}$

(2) for any $x \in \gamma_{x_{-}}, x \neq x_{-}$, the sequence $T^{j} x, j=0,1, \ldots$ converges to $x_{+} \in \Omega$ with $\beta\left(x_{+}\right)<1$,

(3) if the limit of $T^{j} x$ in (2) does not depend on $x$, then $\cup_{j=0} T^{j} \gamma_{x_{-}}=\Gamma_{x_{-}}$is $a C^{1}$ curve segment beginning at $x_{-} \in \Omega, \beta\left(x_{-}\right)>1$, and ending at $x_{+} \in \Omega$, $\beta\left(x_{+}\right)<1$.

We remark here that in this proposition $\Gamma_{x_{-}}$is a $C^{k}$ curve provided that the map $T \in C^{k}$. To study the structure of discrete shock profiles, we need some additional estimates.

Proposition 2.2. Suppose that $x_{ \pm}$in Proposition 2.1 satisfy $\left|x_{ \pm}-x_{0}\right|=O(1) \varepsilon$. Then, for any $x \in \Gamma_{x_{-}}, x \neq x_{ \pm}$,

$$
\begin{gathered}
|\beta(x)-1| \leqq c_{1} \varepsilon, \\
c_{2}|x-T x| \leqq \beta(x)-\beta(T x) \leqq c_{3}|x-T x|, \\
\left|T^{2} x-2 T x+x\right| \leqq c_{4} \varepsilon|T x-x|,
\end{gathered}
$$

where $c_{1}, \ldots, c_{4}$ are constants independent of $x$ and $\varepsilon$.

Proof. It has been shown in Proposition 4.1 of [18] that there is a center manifold $M_{0}$ of $m+1$ dimension, $M_{0} \subset\left\{x:\left|x-x_{0}\right| \leqq O(1) \varepsilon\right\}$ which is in- 
variant under $T$. Furthermore, there are coordinates of $\mathbf{R}^{N},\left(\eta_{1}, \ldots, \eta_{m}, \tau\right.$, $\left.\xi_{m+2}, \ldots, \xi_{N}\right)$, such that

$$
\begin{gathered}
x_{0}=(0,0,0)^{\top}, \\
r=(\underline{r}, 0,0)^{\top}, \quad r \in \mathbf{R}^{m}, \\
\Omega=\left\{(\eta, 0,0)^{\top}: \eta \in \mathbf{R}^{m}\right\}, \\
M_{0}=\left\{(\eta, \tau, 0)^{\top}: \eta \in \mathbf{R}^{m}\right\} \cap\left\{x:\left|x-x_{0}\right| \leqq O(1) \varepsilon\right\}, \\
d T(\eta, \tau, 0)=\left(\begin{array}{cc}
d T_{0}(\eta, \tau) & * \\
0 & *
\end{array}\right)
\end{gathered}
$$

where $T_{0}$ is the restriction of $T$ on $M_{0}$. In these coordinates, $T_{0}$ can be written as

$$
T_{0}(\eta, \tau)=\left(\begin{array}{c}
\eta+\tau \underline{r}+\tau g(\eta, \tau) \\
\tau+\tau h(\eta, \tau)
\end{array}\right)
$$

where $h, g \in C^{1}, h(0,0)=g(0,0)=0$. Clearly, for all $(\eta, \tau) \in M_{0}$,

$$
|g(\eta, \tau)| \leqq O(1) \varepsilon, \quad|h(\eta, \tau)| \leqq O(1) \varepsilon .
$$

Computing the Jacobi matrix for $T_{0}$ directly from (2.9), we get

$$
d T_{0}(\eta, \tau)=\left(\begin{array}{cc}
I_{m} & \underline{r}+g(\eta, \tau) \\
0 & 1+h(\eta, \tau)
\end{array}\right)+\tau \frac{\partial(g, h)}{\partial(\eta, \tau)} .
$$

It follows from (2.8) and condition (v) that, on the center manifold $M_{0}$, the eigenvalues of $d T$ lying in $|z-1| \leqq \delta^{\prime}$ are the same as those of $d T_{0}$, and these eigenvalues are 1 with multiplicity $m$ and $\beta(\eta, \tau)$. The sum of the eigenvalues is equal to the trace of the Jacobi matrix $d T_{0}(\eta, \tau)$, i.e.,

$$
m+\beta(\eta, \tau)=\operatorname{trace} d T_{0}(\eta, \tau)=m+1+h(\eta, \tau)+\tau \operatorname{trace} \frac{\partial(g, h)}{\partial(\eta, \tau)}
$$

or, equivalently,

Consequently,

$$
\beta(\eta, \tau)=1+h(\eta, \tau)+\tau \operatorname{trace} \frac{\partial(g, h)}{\partial(\eta, \tau)} .
$$

$$
\underline{r} \cdot \nabla_{\eta} \beta(\eta, \tau)=\underline{r} \cdot \nabla_{\eta} h(\eta, \tau)+\tau \underline{r} \cdot \nabla_{\eta} \text { trace } \frac{\partial(g, h)}{\partial(\eta, \tau)} .
$$

The entropy condition (2.6) now implies that

$$
r \cdot \nabla_{\eta} h(\eta, \tau)=r \cdot \nabla \beta\left(x_{0}\right)+O(\varepsilon)<0 .
$$

Direct computation using (2.9) yields

$$
\begin{gathered}
\left|T_{0}(\eta, \tau)-(\eta, \tau)\right|=\tau(|\underline{r}|+O(\varepsilon)), \\
\beta\left(T_{0}(\eta, \tau)\right)-\beta(\eta, \tau)=\tau\left(r \cdot \nabla \beta\left(x_{0}\right)+O(\varepsilon)\right), \\
\left|T_{0}^{2}(\eta, \tau)-2 T_{0}(\eta, \tau)+(\eta, \tau)\right| \leqq O(1) \varepsilon \tau,
\end{gathered}
$$

which in turn gives estimates (2.7). 
Proposition 2.3. Suppose that $x_{ \pm}$in Proposition 2.1 satisfy $\left|x_{ \pm}-x_{0}\right|=O(1) \varepsilon$. Then, for any $x \in \Gamma_{x_{-}}, x \neq x_{ \pm}$, and $j=0, \pm 1, \ldots$,

$$
\begin{gathered}
c_{1} \varepsilon^{2} e^{-c_{2} \varepsilon|j|} \leqq \beta\left(T^{j} x\right)-\beta\left(T^{j+1} x\right) \leqq c_{3} \varepsilon^{2} e^{-c_{4} \varepsilon|j|}, \\
c_{5} \varepsilon e^{-c_{6} \varepsilon|j|} \leqq\left|T^{j} x-x_{ \pm}\right| \leqq c_{7} \varepsilon e^{-c_{8} \varepsilon|j|}
\end{gathered}
$$

where $c_{1}, \ldots, c_{8}$ are constants independent of $\varepsilon$ and $j$.

Proof. It is shown in [18] that there is a surface patch $\Sigma$ in the center manifold $M_{0}$ such that $h(\eta, \tau)=0$ on $\Sigma$. The curve $\Gamma_{x_{-}}$joins two points $x_{-}$and $x_{+}$ and pierces $\Sigma$; see Figure 1. We denote the intersection point of $\Gamma_{x_{-}}$with $\Sigma$ as $\left(\eta_{0}, \tau_{0}\right)$, and set

$$
\left(\eta_{j}, \tau_{j}\right)=T^{j}\left(\eta_{0}, \tau_{0}\right)
$$

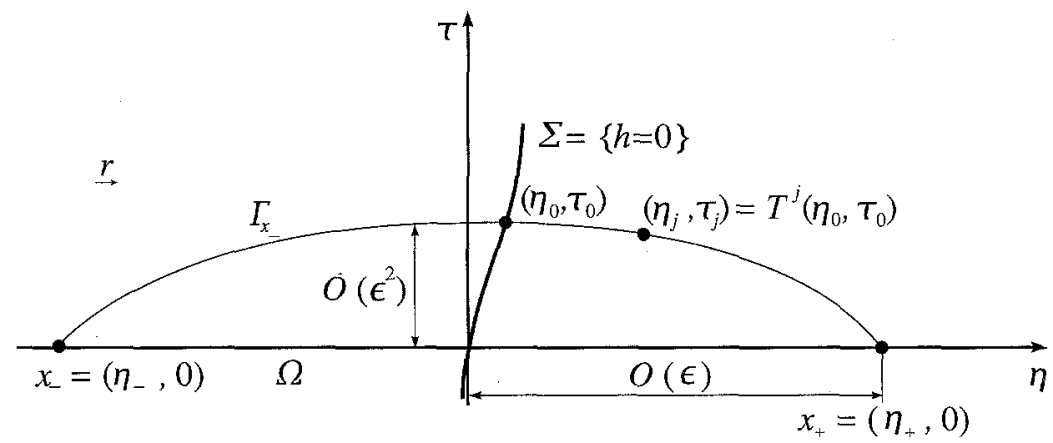

Figure 1. Center manifold construction.

Rewriting (2.9) as

$$
\begin{aligned}
\eta_{j+1} & =\eta_{j}+\tau_{j} \underline{r}+\tau_{j} g\left(\eta_{j}, \tau_{j}\right), \\
\tau_{j+1} & =\tau_{j}+\tau_{j} h\left(\eta_{j}, \tau_{j}\right),
\end{aligned}
$$

we first show that

$$
\left|\tau_{0}\right| \leqq O(1) \varepsilon^{2} \text {. }
$$

By the uniqueness and symmetry of $\Gamma_{x_{-}}$, it suffices to show that for the initial point $x=\left(\eta_{0}, \tau_{0}\right) \in \Sigma$ with $\tau_{0}=O(1) \varepsilon^{2}, T_{0}^{j}\left(\eta_{0}, \tau_{0}\right)$ hits $\Omega$ on the positive $\eta$-axis at a distance of order $O(\varepsilon) 1$ from the origin (i.e., $x_{+}=\left(\eta_{+}, 0\right)$ and $\left.r \cdot \eta_{+}=O(1) \varepsilon\right)$. Since $h\left(\eta_{j}, \tau_{j}\right)<0$ for $j \geqq 1$ and $\left|h\left(\eta_{j}, \tau_{j}\right)\right| \leqq O(1) \varepsilon$, we have

Defining

$$
\tau_{j+1} \geqq \tau_{j}-O(1) \varepsilon \tau_{j} \geqq \tau_{j}-O(1) \varepsilon^{3} .
$$

we get

$$
j_{0}=\left[\frac{1}{2} \frac{\tau_{0}}{O(1) \varepsilon^{3}}\right]=\left[\frac{O(1)}{\varepsilon}\right],
$$

$$
\tau_{j_{0}} \geqq \tau_{0}-j_{0} O(1) \varepsilon^{3}=\tau_{0}-\frac{1}{2} \tau_{0}=\frac{1}{2} \tau_{0} .
$$

Taking the scalar product of $\underline{r}$ with (2.15) leads to

$$
\underline{r} \cdot \eta_{j+1}=\underline{r} \cdot \eta_{j}+\tau_{j}(|\underline{r}|+O(\varepsilon)) .
$$


Summing from 1 to $j_{0}$ yields

$$
\underline{r} \cdot \eta_{j_{0}} \geqq r \cdot \eta_{0}+O(1) j_{0} \tau_{j_{0}} \geqq \underline{r} \cdot \eta_{0}+\frac{1}{2} O(1) j_{0} \tau_{0}=O(1) \varepsilon .
$$

Since $r \cdot \eta_{j}$ is increasing, by using the invariant region, we obtain

$$
\underline{r} \cdot \lim _{j \rightarrow \infty} \eta_{j}=O(1) \varepsilon
$$

which proves (2.16).

Now, we use (2.16) to derive $(2.14 \mathrm{a}, \mathrm{b})$. Summing (2.15) from 0 to $j$ gives

$$
\left(\eta_{j}, \tau_{j}\right)-\left(\eta_{0}, \tau_{0}\right)=\sum_{i=0}^{j} \tau_{i}((r, 0)+O(1) \varepsilon)
$$

This, together with (2.17), implies that for $j \geqq j_{0}$,

$$
\left(\eta_{j}, \tau_{j}\right)-\left(\eta_{0}, \tau_{0}\right)=O(1) \varepsilon(\underline{r}, 0)+O(1) \varepsilon^{2} .
$$

Consequently, for $j \geqq j_{0}$ we have

$$
\begin{aligned}
h\left(\eta_{j}, \tau_{j}\right) & =h\left(\eta_{0}, \tau_{0}\right)+\nabla h\left(\eta_{*}, \tau_{*}\right)\left(\left(\eta_{j}, \tau_{j}\right)-\left(\eta_{0}, \tau_{0}\right)\right) \\
& =O(1) \varepsilon \underline{r} \cdot \nabla_{\eta} h(0,0)+O(1) \varepsilon^{2}=-O(1) \varepsilon .
\end{aligned}
$$

In (2.20), the constant $O(1)$ is positive due to the entropy condition (2.6). Plugging (2.20) into (2.15) gives

$$
\tau_{j+1}=\tau_{j}(1-O(1) \varepsilon) .
$$

Therefore,

$$
\tau_{j}=\tau_{j_{0}} e^{-O(1) \varepsilon\left|j-j_{0}\right|}=O(1) \varepsilon^{2} e^{-O(1) \varepsilon|j|} .
$$

Direct computation using (2.15) leads to

$$
\beta\left(\eta_{j+1}, \tau_{j+1}\right)-\beta\left(\eta_{j}, \tau_{j}\right)=\tau_{j}\left(r \cdot \nabla \beta\left(x_{0}\right)+O(\varepsilon)\right),
$$

so

$$
\beta\left(T^{j} x\right)-\beta\left(T^{j+1} x\right)=O(1) \varepsilon^{2} e^{-O(1) \varepsilon|j|} .
$$

This proves (2.14a). Next, letting $j$ tend to $+\infty$, we have

$$
\left(\eta_{+}, 0\right)-\left(\eta_{j}, \tau_{j}\right)=\sum_{i=j}^{\infty} \tau_{i}((\underline{r}, 0)+O(1) \varepsilon)=O(1) \varepsilon e^{-O(1) \varepsilon|j|}((\underline{r}, 0)+O(1) \varepsilon)
$$

or

$$
\left|\left(\eta_{+}, 0\right)-\left(\eta_{j}, \tau_{j}\right)\right|=O(1) \varepsilon e^{-O(1) \varepsilon|j|},
$$

which yields (2.14b). The proof of Proposition 2.3 is complete.

Now, we use the refined center manifold theorems and Propositions 2.2 and 2.3 , to show the strict monotonicity of the principal characteristic speed at the discrete shock profile and the asymptotic behavior of the discrete shock profiles. These properties are crucial to our stability analysis. 
Theorem 2.2. Under the assumption of Theorem 2.2, the discrete shock profiles $\phi_{j}$ of (2.2) also satisfy, for all $j=0, \pm 1, \ldots$,

$$
\begin{gathered}
\lambda_{k}\left(\phi_{j}\right)>\lambda_{k}\left(\phi_{j+1}\right), \\
\left|\lambda_{p}\left(\phi_{j}\right)-s\right| \leqq c_{1} \varepsilon \\
c_{2}\left|\phi_{j}-\phi_{j+1}\right| \leqq \lambda_{k}\left(\phi_{j}\right)-\lambda_{k}\left(\phi_{j+1}\right) \leqq c_{3}\left|\phi_{j}-\phi_{j+1}\right|, \\
\nabla f\left(\phi_{j}\right)\left(\phi_{j+1}-\phi_{j}\right)=s\left(\phi_{j+1}-\phi_{j}\right)+O(\varepsilon)\left|\phi_{j+1}-\phi_{j}\right|, \\
\left|\phi_{j+1}-2 \phi_{j}+\phi_{j-1}\right| \leqq c_{4} \varepsilon\left|\phi_{j+1}-\phi_{j}\right|, \\
c_{5} \varepsilon^{2} e^{-c_{6} \varepsilon|j|} \leqq \lambda_{k}\left(\phi_{j}\right)-\lambda_{k}\left(\phi_{j+1}\right) \leqq c_{7} \varepsilon^{2} e^{-c_{8} \varepsilon|j|}, \\
c_{9} \varepsilon e^{-c_{10} \varepsilon|j|} \leqq\left|\phi_{j}-u_{ \pm}\right| \leqq c_{11} \varepsilon e^{-c_{12} \varepsilon|j|}
\end{gathered}
$$

where $c_{1}, \ldots, c_{12}$ are positive constants independent of $\varepsilon$ and $j$.

Proof. Note that the derivation of the equality (3.25) in [18] implies that $0=\left(s-\lambda_{k}(u)\right) \lambda q+\frac{1}{2} q \kappa(u) \ln \beta(u)+g_{1}(u) \ln ^{2} \beta(u)+g_{2}(u)\left(s-\lambda_{k}(u)\right)^{2}$ where $g_{1}$ and $g_{2}$ are smooth functions and $\kappa(u)$ is given by (2.16). Taking $u$ first as $\phi_{j}$ and then as $\phi_{j-1}$ in this equality and substracting the resulting equations, we arrive at

$$
\begin{aligned}
\lambda_{k}\left(\phi_{j}\right)-\lambda_{k}\left(\phi_{j+1}\right) & =\frac{1}{2 \lambda} \kappa\left(\phi_{j}\right)\left(\ln \beta\left(\phi_{j}\right)-\ln \beta\left(\phi_{j+1}\right)\right)+O(\varepsilon)\left|\phi_{j}-\phi_{j+1}\right| \\
& =(1+O(\varepsilon)) \frac{1}{2 \lambda} \kappa\left(\phi_{0}\right)\left(\beta\left(\phi_{j}\right)-\beta\left(\phi_{j+1}\right)\right)+O(\varepsilon)\left|\phi_{j}-\phi_{j+1}\right| \\
& =(1+O(\varepsilon)) \frac{1}{2 \lambda} \kappa\left(\phi_{0}\right)\left(\beta\left(\phi_{j}\right)-\beta\left(\phi_{j+1}\right)\right),
\end{aligned}
$$

where $(2.7 \mathrm{a})$ has been used in the last equality. From $(2.7 \mathrm{a}-\mathrm{c})$ and $(2.14 \mathrm{a}, \mathrm{b})$, we know that $\phi_{j}$ satisfies $(2.24 \mathrm{a}-\mathrm{g})$. This completes the proof of the theorem.

Corollary 2.1. Let $\phi_{j}^{n}$ be the discrete shock profiles (1.6) of the Lax-Friedrichs scheme (1.5) connecting $u_{-}$and $u_{+}$. Suppose $u_{ \pm}$satisfy (1.2), $\varepsilon=\left|u_{-}-u_{+}\right|$is suitably small, and $\lambda s=p / q$ for some relatively prime integers $p$ and $q$. Then for all $j=0, \pm 1, \ldots$ and $n=0,1, \ldots$, the $\phi_{j}^{n}$ satisfy

$$
\begin{gathered}
\lambda_{k}\left(\phi_{j}^{n}\right)>\lambda_{k}\left(\phi_{j+1}^{n}\right), \\
\left|\lambda_{p}\left(\phi_{j}^{n}\right)-s\right| \leqq c_{1} \varepsilon, \\
c_{2}\left|\phi_{j}^{n}-\phi_{j+1}^{n}\right| \leqq \lambda_{k}\left(\phi_{j}^{n}\right)-\lambda_{k}\left(\phi_{j+1}^{n}\right) \leqq c_{3}\left|\phi_{j}^{n}-\phi_{j+1}^{n}\right|, \\
\nabla f\left(\phi_{j}^{n}\right)\left(\phi_{j+1}^{n}-\phi_{j}^{n}\right)=s\left(\phi_{j+1}^{n}-\phi_{j}^{n}\right)+O(\varepsilon)\left|\phi_{j+1}^{n}-\phi_{j}^{n}\right|,
\end{gathered}
$$




$$
\begin{gathered}
\left|\phi_{j+1}^{n}-2 \phi_{j}^{n}+\phi_{j-1}^{n}\right| \leqq c_{4} \varepsilon\left|\phi_{j+1}^{n}-\phi_{j}^{n}\right|, \\
c_{5} \varepsilon^{2} e^{-c_{6} \varepsilon|j-\lambda s n|} \leqq \lambda_{k}\left(\phi_{j}^{n}\right)-\lambda_{k}\left(\phi_{j+1}^{n}\right) \leqq c_{7} \varepsilon^{2} e^{-c_{8} \varepsilon|j-\lambda s n|}, \\
c_{9} \varepsilon e^{-c_{10} \varepsilon|j-\lambda s n|} \leqq\left|\phi_{j}^{n}-u_{ \pm}\right| \leqq c_{11} \varepsilon e^{-c_{12} \varepsilon|j-\lambda s n|}
\end{gathered}
$$

where $c_{1}, \ldots, c_{12}$ are positive constants independent of $\varepsilon, j$ and $n$.

Proof. Since the hypotheses of Theorem 2.2 hold in Corollary 2.1, we know that $\phi_{j}^{0}$ satisfies (2.26). Directly from (1.6), one can easily verify that, for $n=0, \ldots, q-1, \phi_{j}^{n}$ satisfies (2.26). Since $\phi_{j}^{n q+l}=\phi_{j-n p}^{l}$ we know that $\phi_{j}^{n}$ also satisfies $(2.26 \mathrm{a}-\mathrm{g})$ for all $n \geqq 0$.

\section{3. $L^{2}$-Stability Analysis for Single Shocks}

In this section we prove the first part of Theorem 1.1, the $L^{2}$-stability of single discrete shocks. We first reformulate the problem as follows. Let $u_{j}^{n}$ be a solution of the Lax-Friedrichs scheme $(1.5 \mathrm{a})$ with initial data $u_{j}^{0}$ satisfying (1.7a), which is assumed to exist up to $n \leqq n_{1}<+\infty$. Denote by $\phi_{j}^{n}$ the single discrete shock profile in the $k$-field whose existence has been proved in Section 2.

Setting

$$
\bar{v}_{j}^{n}=\sum_{i=-\infty}^{j}\left(u_{i}^{n}-\phi_{i}^{n}\right),
$$

subtracting (1.6a) from (1.5a), summing the resulting expression from $-\infty$ to $j$, and using some manipulations we obtain

$$
\begin{aligned}
\bar{v}_{j}^{n+1} & -\bar{v}_{j}^{n}+\frac{\lambda}{2} \nabla f\left(\phi_{j+1}^{n}\right)\left(\bar{v}_{j+1}^{n}-\bar{v}_{j}^{n}\right)+\frac{\lambda}{2} \nabla f\left(\phi_{j}^{n}\right)\left(\bar{v}_{j}^{n}-\bar{v}_{j-1}^{n}\right) \\
& +\frac{\lambda}{2} Q\left(\phi_{j+1}^{n}, \bar{v}_{j+1}^{n}-\bar{v}_{j}^{n}\right)+\frac{\lambda}{2} Q\left(\phi_{j}^{n}, \bar{v}_{j}^{n}-\bar{v}_{j-1}^{n}\right)=\frac{v}{2}\left(\bar{v}_{j+1}^{n}-2 \bar{v}_{j}^{n}+\bar{v}_{j-1}^{n}\right)
\end{aligned}
$$

where

$$
Q(\phi, u-\phi)=f(u)-f(\phi)-\nabla f(\phi)(u-\phi)
$$

satisfies the estimate

$$
|Q(\phi, u-\phi)| \leqq O(1)|u-\phi|^{2}
$$

for $u$ on any bounded set. Using the notations

$L_{j}^{n}=L\left(\phi_{j}^{n}\right), \quad \Lambda_{j}^{n}=\Lambda\left(\phi_{j}^{n}\right), \quad R_{j}^{n}=R\left(\phi_{j}^{n}\right), \quad \theta_{j}^{n}=Q\left(\phi_{j}^{n}, \bar{v}_{j}^{n}-\bar{v}_{j-1}^{n}\right)$,

we rewrite equations (3.2) in terms of characteristic variables

$$
v_{j}^{n}=L_{j}^{n} \bar{v}_{j}^{n}
$$


as

$$
\begin{gathered}
v_{j}^{n+1}-v_{j}^{n}+\frac{\lambda}{2} \Lambda_{j+1}^{n}\left(v_{j+1}^{n}-v_{j}^{n}\right)+\frac{\lambda}{2} \Lambda_{j}^{n}\left(v_{j}^{n}-v_{j-1}^{n}\right)-\frac{v}{2}\left(v_{j+1}^{n}-2 v_{j}^{n}+v_{j-1}^{n}\right) \\
=\left(L_{j}^{n+1}-L_{j}^{n}+\frac{\lambda}{2} \Lambda_{j}^{n}\left(L_{j+1}^{n}-L_{j-1}^{n}\right)\right) R_{j}^{n} v_{j}^{n}+e_{j}^{n},
\end{gathered}
$$

where

$$
\begin{aligned}
e_{j}^{n}= & \left(L_{j}^{n+1}-L_{j}^{n}\right) R_{j}^{n+1}\left(v_{j}^{n+1}-v_{j}^{n}\right)+\left(L_{j}^{n+1}-L_{j}^{n}\right)\left(R_{j}^{n+1}-R_{j}^{n}\right) v_{j}^{n} \\
& +\frac{\lambda}{2}\left(\Lambda_{j+1}^{n}-\Lambda_{j}^{n}\right)\left(L_{j+1}^{n}-L_{j}^{n}\right) R_{j}^{n} v_{j}^{n}-\frac{\lambda}{2} L_{j}^{n}\left(R_{j+1}^{n}-R_{j}^{n}\right) \Lambda_{j+1}^{n}\left(v_{j+1}^{n}-v_{j}^{n}\right) \\
& +\frac{\lambda}{2} L_{j}^{n}\left(R_{j+1}^{n}-R_{j}^{n}\right) \Lambda_{j+1}^{n}\left(L_{j+1}^{n}-L_{j}^{n}\right) R_{j}^{n} v_{j}^{n} \\
& -\frac{v}{2} \Lambda_{j}^{n}\left(L_{j}^{n}-L_{j-1}^{n}\right) R_{j-1}^{n}\left(v_{j}^{n}-v_{j-1}^{n}\right) \\
& -\frac{\lambda}{2} \Lambda_{j}^{n}\left(L_{j}^{n}-L_{j-1}^{n}\right)\left(R_{j}^{n}-R_{j-1}^{n}\right) v_{j}^{n}+\frac{v}{2} L_{j}^{n}\left(R_{j+1}^{n}-R_{j}^{n}\right)\left(v_{j+1}^{n}-v_{j}^{n}\right) \\
& +\frac{v}{2} L_{j}^{n}\left(R_{j}^{n}-R_{j-1}^{n}\right)\left(v_{j}^{n}-v_{j-1}^{n}\right)+\frac{v}{2} L_{j}^{n}\left(R_{j+1}^{n}-2 R_{j}^{n}+R_{j-1}^{n}\right) v_{j}^{n} \\
& -\frac{\lambda}{2} L_{j}^{n}\left(\theta_{j+1}^{n}+\theta_{j}^{n}\right) .
\end{aligned}
$$

Thus, to prove the first part of Theorem 1.1, we need to derive a basic a priori $L^{2}$-estimate on the solution of (3.7). Taking the scalar product of equations (3.7) with $2 v_{j}^{n}$ and using summation by parts, we obtain

$$
\begin{aligned}
& 2 \sum_{j} v_{j}^{n} \cdot\left({ }_{j}^{n+1}-v_{j}^{n}\right)+\lambda \sum_{j} v_{j}^{n} \cdot\left(\Lambda_{j}^{n}-\Lambda_{j+1}^{n}\right) v_{j}^{n}+v \sum_{j}\left|v_{j+1}^{n}-v_{j}^{n}\right|^{2} \\
& \quad=2 \sum_{j} v_{j}^{n} \cdot\left(L_{j}^{n+1}-L_{j}^{n}+\frac{\lambda}{2} \Lambda_{j}^{n}\left(L_{j+1}^{n}-L_{j-1}^{n}\right)\right) R_{j}^{n} v_{j}^{n}+2 \sum_{j} v_{j}^{n} \cdot e_{j}^{n} .
\end{aligned}
$$

Using the identity

$$
\left|v_{j}^{n+1}\right|^{2}-\left|v_{j}^{n}\right|^{2}=2 v_{j}^{n} \cdot\left(v_{j}^{n+1}-v_{j}^{n}\right)+\left|v_{j}^{n+1}-v_{j}^{n}\right|^{2}
$$

in (3.9), we arrive at

$$
\begin{aligned}
\sum_{j}\left|v_{j}^{n+1}\right|^{2}- & \sum_{j}\left|v_{j}^{n}\right|^{2}+\lambda \sum_{j} v_{j}^{n} \cdot\left(\Lambda_{j}^{n}-\Lambda_{j+1}^{n}\right) v_{j}^{n}+v \sum_{j}\left|v_{j+1}^{n}-v_{j}^{n}\right|^{2} \\
= & 2 \sum_{j} v_{j}^{n} \cdot\left(L_{j}^{n+1}-L_{j}^{n}+\frac{\lambda}{2} \Lambda_{j}^{n}\left(L_{j+1}^{n}-L_{j-1}^{n}\right)\right) R_{j}^{n} v_{j}^{n} \\
& +\sum_{j}\left|v_{j}^{n+1}-v_{j}^{n}\right|^{2}+2 \sum_{j} v_{j}^{n} \cdot e_{j}^{n} .
\end{aligned}
$$


Set

$$
M\left(n_{1}\right)=\sup _{n \leqq n_{1}}\left(\sum_{j}\left|v_{j}^{n}\right|^{2}\right)^{1 / 2}
$$

and assume a priori that $M\left(n_{1}\right)$ is small. Clearly we have

$$
\sup _{(j, n), n \leqq n_{1}}\left|v_{j}^{n}\right| \leqq M\left(n_{1}\right) \text {. }
$$

We now estimate each term on the right-hand side of (3.11). It follows from (1.6) and Corollary 2.1 that

$$
\begin{aligned}
L_{j}^{n+1}-L_{j}^{n}+\frac{\lambda s}{2}\left(L_{j+1}^{n}-L_{j-1}^{n}\right)= & (1+O(\varepsilon)) \nabla L\left(\phi_{j}^{n}\right) \\
& \times\left(\phi_{j}^{n+1}-\phi_{j}^{n}+\frac{\lambda s}{2}\left(\phi_{j+1}^{n}-\phi_{j-1}^{n}\right)\right) \\
= & O(\varepsilon)\left(\lambda_{k, j}^{n}-\lambda_{k, j+1}^{n}\right) .
\end{aligned}
$$

Using the identity

$$
\begin{aligned}
L_{j}^{n+1}-L_{j}^{n}+\frac{\lambda}{2} & \Lambda_{j}^{n}\left(L_{j+1}^{n}-L_{j-1}^{n}\right) \\
& =L_{j}^{n+1}-L_{j}^{n}+\frac{\lambda s}{2}\left(L_{j+1}^{n}-L_{j-1}^{n}\right)+\frac{\lambda}{2}\left(\Lambda_{j}^{n}-s\right)\left(L_{j+1}^{n}-L_{j-1}^{n}\right),
\end{aligned}
$$

and (3.14), we obtain

$$
\begin{aligned}
& 2 v_{j}^{n} \cdot\left(L_{j}^{n+1}-L_{j}^{n}+\frac{\lambda}{2} \Lambda_{j}^{n}\left(L_{j+1}^{n}-L_{j-1}^{n}\right)\right) R_{j}^{n} v_{j}^{n} \\
& \quad \leqq \frac{\lambda}{8}\left(\lambda_{k, j}^{n}-\lambda_{k, j+1}^{n}\right)\left(v_{k, j}^{n}\right)^{2}+O(1)\left(\lambda_{k, j}^{n}-\lambda_{k, j+1}^{n}\right) \sum_{\mu \neq k}\left(v_{\mu, j}^{n}\right)^{2} .
\end{aligned}
$$

It follows from equation (3.7) that

$$
\begin{aligned}
\left|v_{j}^{n+1}-v_{j}^{n}\right| \leqq & \frac{1}{2}\left(\lambda|\Lambda|_{L^{\infty}}+v+O(\varepsilon)+M\left(n_{1}\right)\right)\left(\left|v_{j+1}^{n}-v_{j}^{n}\right|+\left|v_{j}^{n}-v_{j-1}^{n}\right|\right) \\
& +O(1)\left|\left(\lambda_{k, j}^{n}-\lambda_{k, j+1}^{n}\right) v_{j}^{n}\right|
\end{aligned}
$$

where we have used the bound

$$
\left|\theta_{j}^{n}\right| \leqq O(1)\left(\left|v_{j}^{n}-v_{j+1}^{n}\right|^{2}+\left(\lambda_{k, j}^{n}-\lambda_{k, j+1}^{n}\right)^{2}\left|v_{j}^{n}\right|^{2}\right)
$$

(see 3.3)). Consequently

$$
\begin{aligned}
\sum_{j}\left|v_{j}^{n+1}-v_{j}^{n}\right|^{2} \leqq & \frac{\lambda}{8} \sum_{j}\left(\lambda_{k, j}^{n}-\lambda_{k, j+1}^{n}\right)\left|v_{j}^{n}\right|^{2} \\
& +\left(\left(\lambda|\Lambda|_{L^{\infty}}+v\right)^{2}+O(\varepsilon)+O(1) M\left(n_{1}\right)\right) \sum_{j}\left|v_{j+1}^{n}-v_{j}^{n}\right|^{2}
\end{aligned}
$$

where we have used Corollary 2.1. 
Next, using (3.8), (3.17) and Corollary 2.1, we get after some detailed manipulations that

$$
\begin{aligned}
\sum_{j} v_{j}^{n} \cdot e_{j}^{n} \leqq & \frac{\lambda}{8} \sum_{j}\left(\lambda_{k, j}^{n}-\lambda_{k, j+1}^{n}\right)\left|v_{j}^{n}\right|^{2}+O(\varepsilon) \sum_{j}\left|v_{j}^{n+1}-v_{j}^{n}\right|^{2} \\
& +\left(O(\varepsilon)+O(1) M\left(n_{1}\right)\right) \sum_{j}\left|v_{j+1}^{n}-v_{j}^{n}\right|^{2}
\end{aligned}
$$

In fact, two typical terms involved in establishing (3.19) can be estimated as follows:

$$
\begin{aligned}
&\left|v_{j}^{n} \cdot\left(L_{j}^{n+1}-L_{j}^{n}\right) R_{j}^{n+1}\left(v_{j}^{n+1}-v_{j}^{n}\right)\right| \leqq O(1)\left(\lambda_{k, j}^{n}-\lambda_{k, j+1}^{n}\right)\left|v_{j}^{n} \cdot\left(v_{j}^{n+1}-v_{j}^{n}\right)\right| \\
& \leqq \frac{\lambda}{16}\left(\lambda_{k, j}^{n}-\lambda_{k, j+1}^{n}\right)\left|v_{j}^{n}\right|^{2}+O(\varepsilon)\left|v_{j}^{n+1}-v_{j}^{n}\right|^{2}, \\
&\left|v_{j}^{n} \cdot L_{j}^{n} \theta_{j}^{n}\right| \leqq O(1)\left|v_{j}^{n}\right|\left|v_{j}^{n}-v_{j+1}^{n}\right|^{2}+O(\varepsilon)\left(\lambda_{k, j}^{n}-\lambda_{k, j+1}^{n}\right)\left|v_{j}^{n}\right|^{2} \\
& \leqq \\
& \leqq
\end{aligned}
$$

Estimates (3.15), (3.18) and (3.19), together with (3.11), yield

$$
\begin{aligned}
\sum_{j}\left|v_{j}^{n+1}\right|^{2}- & \sum_{j}\left|v_{j}^{n}\right|^{2}+\frac{\lambda}{4} \sum_{j}\left(\lambda_{k, j}^{n}-\lambda_{k, j+1}^{n}\right)\left|v_{k, j}^{n}\right|^{2} \\
& +\left(v-\left(\lambda|\Lambda|_{L^{\infty}}+v\right)^{2}-O(\varepsilon)-O(1) M\left(n_{1}\right)\right) \sum_{j}\left|v_{j+1}^{n}-v_{j}^{n}\right|^{2} \\
\leqq & O(1) \sum_{j}\left(\lambda_{k, j}^{n}-\lambda_{k, j+1}^{n}\right) \sum_{\mu \neq k}\left|v_{\mu, j}^{n}\right|^{2} .
\end{aligned}
$$

The waves in the transversal directions are bounded by the last term on the right-hand side of (3.20), which is estimated by the following proposition.

Proposition 3.1 (Estimate on the transversal waves).

$$
\begin{aligned}
\sum_{j, n \leqq n_{2}}\left(\lambda_{k, j}^{n}-\lambda_{k, j+1}^{n}\right) \sum_{\mu \neq k}\left(v_{\mu, j}^{n}\right)^{2} & \leqq \\
& O(\varepsilon) \sum_{j}\left|v_{j}^{0}\right|^{2}+O(\varepsilon) \sum_{j, n \leqq n_{2}}\left(\lambda_{k, j}^{n}-\lambda_{k, j+1}^{n}\right)\left(v_{k, j}^{n}\right)^{2} \\
& +O(\varepsilon) \sum_{j, n \leqq n_{2}}\left|v_{j+1}^{n}-v_{j}^{n}\right|^{2} .
\end{aligned}
$$

We prove Proposition 3.1 at the end of this section. Since $v<1$, by taking $\varepsilon$ and $\lambda$ suitably small and applying Proposition 3.1, we prove the following basic a priori estimate.

Proposition 3.2 (A priori estimate). Let $v_{j}^{n}$ be a solution of (3.7) for $n \leqq n_{1}$. Then there exists a positive constant $C$ independent of $n_{1}$ and $\varepsilon$ such 
that

$\sum_{j}\left|v_{j}^{n_{2}}\right|^{2}+\sum_{j, n \leqq n_{2}}\left|v_{j}^{n}-v_{j+1}^{n}\right|^{2}+\sum_{j, n \leqq n_{2}}\left|\lambda_{k, j}^{n}-\lambda_{k, j+1}^{n}\right|\left|v_{k, j}^{n}\right|^{2} \leqq C \sum_{j}\left|v_{j}^{0}\right|^{2}$

for all $n_{2} \leqq n_{1}$, provided that $\varepsilon, \lambda$ and $M\left(n_{1}\right)$ are suitably small.

In Proposition 3.1 and in what follows, for simplicity of presentation we use the notation

$$
\sum_{j, n \leqq n_{2}}=\sum_{j} \sum_{n \leqq n_{2}}
$$

Since (3.7) is a discrete uniformly parabolic system, it follows from Proposition 3.1 and the standard continuity argument [23] that the following proposition holds.

Proposition 3.3. Assume that $\varepsilon$ and $M(0)$ are suitably small. Then problem (3.5) has a unique global solution $v_{j}^{n}$ satisfying

$\sup _{n} \sum_{j}\left|v_{j}^{n}\right|^{2}+\sum_{j, n}\left|v_{j}^{n}-v_{j+1}^{n}\right|^{2}+\sum_{j, n}\left|\lambda_{k, j}^{n}-\lambda_{k, j+1}^{n}\right|\left|v_{k, j}^{n}\right|^{2} \leqq C M^{2}(0)$

for any $n \geqq 0$, where $C$ is a positive constant independent of $n$ and $j$.

With Proposition 3.3 at hand, we can obtain the $L^{2}$-asymptotic stability result quite easily.

Proof of the First Part of Theorem 1.1. First we show that condition (1.7) implies that $M(0)$ is small. Here we give a proof under the condition that

$$
\sum_{j=-\infty}^{\infty}\left(1+j^{2}\right)^{\alpha}\left|u_{j}^{0}-\phi_{j}^{0}\right|^{2} \leqq c_{2}
$$

for any given constant $\alpha>1$. The proof for the case $\alpha=1$ requires a discrete version of a weighted Poincaré inequality; for details, see [9]. Applying the Hölder inequality to

gives

$$
\bar{v}_{j}^{0}=\sum_{i=-\infty}^{j}\left(u_{i}^{0}-\phi_{i}^{0}\right)
$$

$$
\left|\bar{v}_{j}^{0}\right|^{2} \leqq \sum_{i=-\infty}^{j}\left(1+i^{2}\right)^{\alpha}\left|u_{i}^{0}-\phi_{i}^{0}\right|^{2} \sum_{i=-\infty}^{j}\left(1+i^{2}\right)^{-\alpha} \leqq c_{2} \sum_{i=-\infty}^{j}\left(1+i^{2}\right)^{-\alpha}
$$

Therefore,

$$
\sum_{j=-\infty}^{0}\left|\bar{v}_{j}^{0}\right|^{2} \leqq c_{2} \sum_{j=-\infty}^{0} \sum_{i=-\infty}^{j}\left(1+i^{2}\right)^{-\alpha} \leqq \frac{c_{2}}{2(\alpha-1)} .
$$

Similarly, applying the Hölder inequality to

$$
\bar{v}_{j}^{0}=\sum_{i=-\infty}^{j}\left(u_{i}^{0}-\phi_{i}^{0}\right)=-\sum_{i=j+1}^{\infty}\left(u_{i}^{0}-\phi_{i}^{0}\right)
$$


and using (1.7a), we get

$$
\sum_{j=1}^{\infty}\left|\bar{v}_{j}^{0}\right|^{2} \leqq \frac{c_{2}}{2(\alpha-1)} .
$$

Combining this with (3.24) yields the desired result:

or, equivalently,

$$
\sum_{j=-\infty}^{\infty}\left|\bar{v}_{j}^{0}\right|^{2} \leqq \frac{c_{2}}{(\alpha-1)}
$$

$$
M(0)=\left(\sum_{j=-\infty}^{\infty}\left|v_{j}^{0}\right|^{2}\right)^{1 / 2} \leqq O(1) \sqrt{c_{2}} .
$$

Thus the hypothesis in Proposition 3.3 is fulfilled under the conditions (1.7). It follows from Proposition 3.3 that there exists a unique global solution $u_{j}^{n}$ to the Lax-Friedrichs scheme (1.5) by virtue of the relation

which follows from (3.1).

$$
u_{j}^{n}=\phi_{j}^{n}+\bar{v}_{j}^{n}-\bar{v}_{j-1}^{n},
$$

Next, we study the asymptotic behavior of the solution $u_{j}^{n}$ to (1.5). It follows from Proposition 3.3 that

which implies

$$
\sum_{n=1}^{\infty}\left(\sum_{j}\left|\bar{v}_{j}^{n}-\bar{v}_{j+1}^{n}\right|^{2}\right)<+\infty,
$$

Using (3.1) again, we have

$$
\lim _{n \rightarrow \infty} \sum_{j}\left|\bar{v}_{j}^{n}-\bar{v}_{j+1}^{n}\right|^{2}=0 .
$$

$$
\lim _{n \rightarrow \infty} \sum_{j}\left|u_{j}^{n}-\phi_{j}^{n}\right|^{2}=\lim _{n \rightarrow \infty} \sum_{j}\left|\bar{v}_{j}^{n}-\bar{v}_{j+1}^{n}\right|^{2}=0,
$$

which is exactly the estimate (1.8). The proof of the first part of Theorem 1.1 is complete.

Finally we prove Proposition 3.1. As we remarked earlier, the estimates on transversal waves are technically quite involved due to wave interactions and the fact that our shocks are non-stationary. We employ a modified version of the "vertical estimate" [5]. The main idea is to relate a sum along time-like discrete segments to a sum over all the grid points in a strip in the space-time plane.

Proof of Proposition 3.1. Suppose that $\mu>k$ (the case $\mu<k$ can be treated similarly). To simplify the presentation, we introduce some notations here. For any fixed space index $j_{0}$, we define the $k$-th time-like grid line originated at $\left(j_{0}, 0\right)$ to be

$J\left(j_{0}\right)=\left\{\left(n, j_{n}\right) \mid j_{n}=j_{0}+(l-1) p\right.$ for $\left.(l-1)|p| \leqq n<l|p|, l=1,2, \ldots\right\}$. 


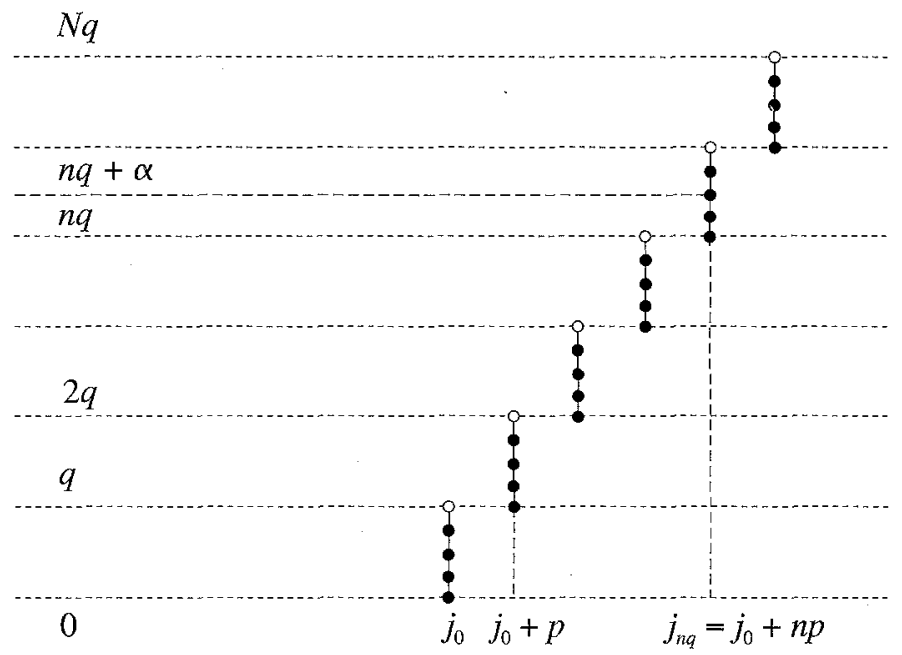

Figure 2. Time-like grid line $J\left(j_{0}\right)$.

See Figure 2. We now prove estimate (3.21). Let $N$ be any given positive integer and $j_{0}$ a given space index. For any integer $n, 0 \leqq n \leqq N q-1$, multiplying the $\mu$-th equation in (3.7) by $2 v_{\mu, j}^{n}$ and summing by parts over $j$ from $-\infty$ to $j_{n}$, we obtain

$$
\begin{aligned}
& \sum_{j=-\infty}^{j_{n}}\left(\left(v_{\mu, j}^{n+1}\right)^{2}-\left(v_{\mu, j}^{n}\right)^{2}\right)+\frac{p}{q}\left(v_{\mu, j_{n}}^{n}\right)^{2}+\lambda\left(\lambda_{\mu, j_{n}}^{n}-s\right)\left(v_{\mu, j_{n}}^{n}\right)^{2} \\
& \leqq \lambda \sum_{j=-\infty}^{j_{n}}\left(\lambda_{\mu, j}^{n}-\lambda_{\mu, j-1}^{n}\right)\left(v_{\mu, j-1}^{n}\right)^{2}-v \sum_{j=-\infty}^{j_{n}}\left(v_{\mu, j}^{n}-v_{\mu, j-1}^{n}\right)^{2} \\
&+\sum_{j=-\infty}^{j_{n}}\left(2\left|v_{j}^{n} \cdot e_{j}^{n}\right|+\left|v_{j}^{n+1}-v_{j}^{n}\right|^{2}\right)+\left(v-\lambda_{\mu, j_{n}+1}^{n}\right) v_{\mu, j_{n}}^{n}\left(v_{\mu, j_{n}+1}^{n}-v_{\mu, j_{n}}^{n}\right) \\
& \leqq O(1) \sum_{j=-\infty}^{\infty}\left(\lambda_{k, j}^{n}-\lambda_{k, j+1}^{n}\right)\left|v_{j}^{n}\right|^{2}+O(1) \sum_{j=-\infty}^{\infty}\left|v_{j+1}^{n}-v_{j}^{n}\right|^{2} \\
&+\left(v-\lambda_{\mu, j_{n}+1}^{n}\right) v_{\mu, j_{n}}^{n}\left(v_{\mu, j_{n}+1}^{n}-v_{\mu, j_{n}}^{n}\right)
\end{aligned}
$$

which, when summed from 0 to $N q-1$, yields

$$
\begin{aligned}
& \sum_{n=0}^{N q-1} \sum_{j=-\infty}^{j_{n}}\left(\left(v_{\mu, j}^{n+1}\right)^{2}-\left(v_{\mu, j}^{n}\right)^{2}\right)+\frac{p}{q} \sum_{n=0}^{N q-1}\left|v_{\mu, j_{n}}^{n}\right|^{2}+\lambda \sum_{n=0}^{N q-1}\left(\lambda_{\mu, j_{n}}^{n}-s\right)\left(v_{\mu, j_{n}}^{n}\right)^{2} \\
& \leqq \\
& O(1) \sum_{n=0}^{N q-1} \sum_{j=-\infty}^{\infty}\left(\lambda_{k, j}^{n}-\lambda_{k, j+1}^{n}\right)\left|v_{j}^{n}\right|^{2}+O(1) \sum_{n=0}^{N q-1} \sum_{j=-\infty}^{\infty}\left|v_{j+1}^{n}-v_{j}^{n}\right|^{2} \\
& \quad+O(1) \sum_{n=0}^{N q-1} v_{\mu, j_{n}}^{n}\left(v_{\mu, j_{n}+1}^{n}-v_{\mu, j_{n}}^{n}\right)
\end{aligned}
$$


We now assert that

$$
\begin{aligned}
& \sum_{n=0}^{N q-1} \sum_{j=-\infty}^{j_{n}}\left(\left(v_{\mu, j}^{n+1}\right)^{2}-\left(v_{\mu, j}^{n}\right)^{2}\right)+\frac{p}{q} \sum_{n=0}^{N q-1}\left(v_{\mu, j_{n}}^{n}\right)^{2} \\
& \quad \geqq \sum_{j=-\infty}^{j_{0}+N p}\left(v_{\mu, j}^{N q}\right)^{2}-\sum_{j=-\infty}^{j_{0}-p}\left(v_{\mu, j}^{0}\right)^{2}-\frac{O(1)}{\delta} \sum_{n=0}^{N q-1} \sum_{j=-\infty}^{\infty}\left|v_{j+1}^{n}-v_{j}^{n}\right|^{2}-\delta \sum_{n=0}^{N-1}\left(v_{\mu, j_{n}}^{n}\right)^{2}
\end{aligned}
$$

where $\delta>0$ will be determined later.

Assuming for a moment that (3.27) holds, we find that for suitably chosen $\delta$

$$
\begin{aligned}
\sum_{n=0}^{N q-1}\left(v_{\mu, j_{n}}^{n}\right)^{2} \leqq & O(1) \sum_{j=-\infty}^{\infty}\left|v_{j}^{0}\right|^{2}+O(1) \sum_{n=0}^{N q-1} \sum_{j=-\infty}^{\infty}\left(\lambda_{k, j}^{n}-\lambda_{k, j+1}^{n}\right)\left|v_{j}^{n}\right|^{2} \\
& +O(1) \sum_{n=0}^{N q-1} \sum_{j=-\infty}^{\infty}\left|v_{j+1}^{n}-v_{j}^{n}\right|^{2}+O(1) \sum_{n=0}^{N q-1}\left|v_{\mu, j_{n}}^{n}\left(v_{\mu, j_{n}+1}^{n}-v_{\mu, j_{n}}^{n}\right)\right|
\end{aligned}
$$

Applying Cauchy's inequality to the last term on the right-hand side of (3.28) gives

$$
\begin{aligned}
\sum_{n=0}^{N q-1}\left(v_{\mu, j_{n}}^{n}\right)^{2} \leqq & O(1) \sum_{j=-\infty}^{\infty}\left|v_{j}^{0}\right|^{2}+O(1) \sum_{n=0}^{N q-1} \sum_{j=-\infty}^{\infty}\left(\lambda_{k, j}^{n}-\lambda_{k, j+1}^{n}\right)\left|v_{j}^{n}\right|^{2} \\
& +O(1) \sum_{n=0}^{N q-1} \sum_{j=-\infty}^{\infty}\left|v_{j+1}^{n}-v_{j}^{n}\right|^{2}
\end{aligned}
$$

We now multiply both sides of (3.29) by $\lambda_{k, j_{0}}^{0}-\lambda_{k, j_{0}+1}^{0}$ and sum over $j_{0}$ from $-\infty$ to $\infty$ to obtain

$$
\begin{aligned}
& \sum_{j_{0}=-\infty}^{\infty}\left(\lambda_{k, j_{0}}^{0}-\lambda_{k, j_{0}+1}^{0}\right) \sum_{n=0}^{N q-1}\left(v_{\mu, j_{n}}^{n}\right)^{2} \\
& \leqq O(\varepsilon) \sum_{j=-\infty}^{\infty}\left|v_{j}^{0}\right|^{2}+O(\varepsilon) \sum_{n=0}^{N q-1} \sum_{j=-\infty}^{\infty}\left(\lambda_{k, j}^{n}-\lambda_{k, j+1}^{n}\right)\left|v_{j}^{n}\right|^{2} \\
&+O(\varepsilon) \sum_{n=0}^{N q-1} \sum_{j=-\infty}^{\infty}\left|v_{j+1}^{n}-v_{j}^{n}\right|^{2}
\end{aligned}
$$

Noting that

$$
\begin{aligned}
\sum_{n=0}^{N q-1} \sum_{j=-\infty}^{\infty}\left(\lambda_{k, j}^{n}-\lambda_{k, j+1}^{n}\right)\left(v_{\mu, j}^{n}\right)^{2} & =\sum_{j_{0}=-\infty}^{\infty} \sum_{n=0}^{N q-1}\left(\lambda_{k, j_{n}}^{n}-\lambda_{k, j_{n}+1}^{n}\right)\left(v_{\mu, j_{n}}^{n}\right)^{2} \\
& \leqq(1+O(\varepsilon)) \sum_{j_{0}=-\infty}^{\infty}\left(\lambda_{k, j_{0}}^{0}-\lambda_{k, j_{0}+1}^{0}\right) \sum_{n=0}^{N q-1}\left(v_{\mu, j_{n}}^{n}\right)^{2},
\end{aligned}
$$


we conclude from (3.30) that

$$
\begin{aligned}
& \sum_{j, n}\left(\lambda_{k}\left(\phi_{k, j}^{n}\right)-\lambda_{k}\left(\phi_{k, j+1}^{n}\right)\right)\left(v_{\mu, j}^{n}\right)^{2} \\
& \quad \leqq O(\varepsilon) \sum_{j=-\infty}^{\infty}\left|v_{j}^{0}\right|^{2}+O(\varepsilon) \sum_{j, n}\left(\lambda_{k, j}^{n}-\lambda_{k, j+1}^{n}\right)\left|v_{j}^{n}\right|^{2} \sum_{j, n}\left|v_{j+1}^{n}-v_{j}^{n}\right|^{2} .
\end{aligned}
$$

Similar analysis shows that (3.31) is still true for $\mu<k$. Therefore, summation over $\mu$ yields

$$
\begin{aligned}
& \sum_{j, n}\left(\lambda_{k, j}^{n}-\lambda_{k, j+1}^{n}\right) \sum_{\mu \neq k}\left(v_{\mu, j}^{n}\right)^{2} \\
& \quad \leqq O(\varepsilon) \sum_{j}\left|v_{j}^{0}\right|^{2}+O(\varepsilon) \sum_{j, n}\left(\lambda_{k, j}^{n}-\lambda_{k, j+1}^{n}\right)\left|v_{j}^{n}\right|^{2}+O(\varepsilon) \sum_{j, n}\left|v_{j+1}^{n}-v_{j}^{n}\right|^{2} .
\end{aligned}
$$

It follows that

$$
\begin{aligned}
\sum_{j, n}\left(\lambda_{k, j}^{n}-\lambda_{k, j+1}^{n}\right) \sum_{\mu \neq k}\left(v_{\mu, j}^{n}\right)^{2} \leqq & O(\varepsilon) \sum_{j}\left|v_{j}^{0}\right|^{2}+O(\varepsilon) \sum_{j, n}\left(\lambda_{k, j}^{n}-\lambda_{k, j+1}^{n}\right)\left(v_{k, j}^{n}\right)^{2} \\
& +O(\varepsilon) \sum_{j, n}\left|v_{j+1}^{n}-v_{j}^{n}\right|^{2} .
\end{aligned}
$$

To complete the proof of Proposition 3.1 we must verify (3.27): Using (3.7) we compute that

$$
\begin{aligned}
& \sum_{n=0}^{N q-1} \sum_{j=-\infty}^{j_{n}}\left(\left(v_{\mu, j}^{n+1}\right)^{2}-\left(v_{\mu, j}^{n}\right)^{2}\right)+\frac{p}{q} \sum_{n=0}^{N q-1}\left(v_{\mu, j_{n}}^{n}\right)^{2} \\
& \quad=\sum_{j=-\infty}^{j_{0}+N p}\left(v_{\mu, j}^{N q}\right)^{2}-\sum_{j=-\infty}^{j_{0}-p}\left(v_{\mu, j}^{0}\right)^{2}+\frac{1}{q} \sum_{n=0}^{N-1}\left(p \sum_{\alpha=0}^{q-1}\left(v_{\mu, j_{n q}}^{n q+\alpha}\right)^{2}-q \sum_{\beta=1}^{p}\left(v_{\mu, j_{n q}+\beta}^{n q}\right) .\right.
\end{aligned}
$$

The last term on the right-hand side of this equality can be estimated as follows:

$$
\begin{aligned}
& \sum_{n=0}^{N-1}\left(p \sum_{\alpha=0}^{q-1}\left(v_{\mu, j_{n q}}^{n q+\alpha}\right)^{2}-q \sum_{\beta=1}^{p}\left(v_{\mu, j_{n q}+j}^{n q}\right)^{2}\right) \\
& =p \sum_{n=0}^{N-1} \sum_{\alpha=0}^{q-1}\left(v_{\mu, j_{n q}}^{n q+\alpha}-v_{\mu, j_{n q}}^{n q}\right)^{2}+2 p \sum_{n=0}^{N-1} \sum_{\alpha=0}^{q-1}\left(v_{\mu, j_{n q}}^{n q+\alpha}-v_{\mu, j_{n q}}^{n q}\right) v_{\mu, j_{n q}}^{n q} \\
& \quad-q \sum_{n=0}^{N-1} \sum_{j=1}^{p}\left(v_{\mu, j_{n q}+j}^{n q}-v_{\mu, j_{n q}}^{n q}\right)^{2}-2 q \sum_{n=0}^{N-1} \sum_{\beta=1}^{p}\left(v_{\mu, j_{n q}+\beta}^{n q}-v_{\mu, j_{n q}}^{n q}\right) v_{\mu, j_{n q}}^{n q} \\
& \leqq \\
& \quad \frac{O(1)}{\delta} \sum_{j, n}\left(v_{\mu, j}^{n}-v_{\mu, j+1}^{n}\right)^{2}+\delta \sum_{n=0}^{N-1}\left(v_{\mu, j_{n q}}^{n q}\right)^{2} \leqq
\end{aligned}
$$




$$
\begin{aligned}
& \leqq \frac{O(1)}{\delta} \sum_{j, n}\left(v_{\mu, j}^{n}-v_{\mu, j+1}^{n}\right)^{2}+\delta \sum_{n=0}^{N-1} \sum_{\alpha=0}^{q-1}\left(v_{\mu, j_{n q}}^{n q+\alpha}\right)^{2} \\
& =\frac{O(1)}{\delta} \sum_{j, n}\left(v_{\mu, j}^{n}-v_{\mu, j+1}^{n}\right)^{2}+\delta \sum_{n=0}^{N q-1}\left(v_{\mu, j_{n}}^{n}\right)^{2}
\end{aligned}
$$

by the Cauchy inequality. This yields the desired inequality (3.27).

\section{4. $L^{1}$-Stability Analysis for Single Shocks}

We now turn our attention to the $L^{1}$-stability of single discrete shocks. As we remarked in the introduction, $L^{1}$ is the natural norm for the stability of shock waves, but in contrast to scalar conservation laws where $L^{1}$-stability is a simple consequence of the entropy condition, for systems it is quite difficult to obtain an $L^{1}$-estimate due to the interactions of waves from different characteristic families. The main idea of achieving an $L^{1}$-estimate is to exploit the fact that the Lax-Friedrichs scheme is essentially monotonic in each characteristic direction and to employ a carefully chosen weight based on the internal structure of the discrete shock profile. We note that here the characteristic decomposition is essential.

To obtain the $L^{1}$-estimate (1.9) on the solution $u_{j}^{n}$ to the Lax-Friedrichs scheme, we work on the characteristic decomposition introduced in Section 3. We first rewrite (3.7) as

$$
\begin{array}{r}
v_{j}^{n+1}-v_{j}^{n}+\frac{\lambda}{2} \Lambda_{j+1}^{n}\left(v_{j+1}^{n}-v_{j}^{n}\right)+\frac{\lambda}{2} \Lambda_{j}^{n}\left(v_{j}^{n}-v_{j-1}^{n}\right)-\frac{v}{2}\left(v_{j+1}^{n}-2 v_{j}^{n}+v_{j-1}^{n}\right) \\
=A_{j}^{n} v_{j}^{n}+B_{j}^{n}\left(v_{j+1}^{n}-v_{j}^{n}\right)+C_{j}^{n}\left(v_{j}^{n}-v_{j-1}^{n}\right)+D_{j}^{n}\left(v_{j}^{n+1}-v_{j}^{n}\right)+\tilde{e}_{j}^{n}
\end{array}
$$

where $A_{j}^{n}, B_{j}^{n}, C_{j}^{n}$ and $D_{j}^{n}$ are matrices given by

$$
\begin{aligned}
A_{j}^{n} & =\left(L_{j}^{n+1}-L_{j}^{n}+\frac{\lambda}{2} \Lambda_{j}^{n}\left(L_{j+1}^{n}-L_{j-1}^{n}\right)\right) R_{j}^{n}, \\
B_{j}^{n} & =\frac{1}{2} L_{j}^{n}\left(R_{j+1}^{n}-R_{j}^{n}\right)\left(v-\lambda \Lambda_{j+1}^{n}\right), \\
C_{j}^{n} & =\frac{1}{2}\left(v+\lambda \Lambda_{j}^{n}\right) L_{j}^{n}\left(R_{j}^{n}-R_{j-1}^{n}\right), \\
D_{j}^{n} & =\left(L_{j}^{n+1}-L_{j}^{n}\right) R_{j}^{n+1},
\end{aligned}
$$

and $\tilde{e}_{j}^{n}$ is a vector given by

$$
\begin{aligned}
& \tilde{e}_{j}^{n}=\left(L_{j}^{n+1}-L_{j}^{n}\right)\left(R_{j}^{n+1}-R_{j}^{n}\right) v_{j}^{n}+\frac{\lambda}{2}\left(\Lambda_{j+1}^{n}-\Lambda_{j}^{n}\right)\left(L_{j+1}^{n}-L_{j}^{n}\right) R_{j}^{n} v_{j}^{n} \\
& +\frac{\lambda}{2} L_{j}^{n}\left(R_{j+1}^{n}-R_{j}^{n}\right) \Lambda_{j+1}^{n}\left(L_{j+1}^{n}-L_{j}^{n}\right) R_{j}^{n} v_{j}^{n}-\frac{\lambda}{2} \Lambda_{j}^{n}\left(L_{j}^{n}-L_{j-1}^{n}\right)\left(R_{j}^{n}-R_{j-1}^{n}\right) v_{j}^{n} \\
& +\frac{v}{2} L_{j}^{n}\left(R_{j+1}^{n}-2 R_{j}^{n}+R_{j-1}^{n}\right) v_{j}^{n}-\frac{\lambda}{2} L_{j}^{n}\left(\theta_{j+1}^{n}+\theta_{j}^{n}\right)
\end{aligned}
$$


It follows from (4.1) that

$$
\begin{aligned}
\left(I-D_{j}^{n}\right)\left(v_{j}^{n+1}-v_{j}^{n}\right)= & \frac{1}{2}\left(v-\lambda \Lambda_{j+1}^{n}+2 B_{j}^{n}\right)\left(v_{j+1}^{n}-v_{j}^{n}\right) \\
& -\frac{1}{2}\left(v+\lambda \Lambda_{j}^{n}-2 C_{j}^{n}\right)\left(v_{j}^{n}-v_{j-1}^{n}\right)+A_{j}^{n} v_{j}^{n}+\tilde{e}_{j}^{n} .
\end{aligned}
$$

This, together with (4.1), gives

$$
\begin{gathered}
v_{j}^{n+1}-v_{j}^{n}+\frac{\lambda}{2} \Lambda_{j+1}^{n}\left(v_{j+1}^{n}-v_{j}^{n}\right)+\frac{\lambda}{2} \Lambda_{j}^{n}\left(v_{j}^{n}-v_{j-1}^{n}\right)-\frac{v}{2}\left(v_{j+1}^{n}-2 v_{j}^{n}+v_{j-1}^{n}\right) \\
=E_{j}^{n} v_{j}^{n}+F_{j}^{n}\left(v_{j+1}^{n}-v_{j}^{n}\right)+G_{j}^{n}\left(v_{j}^{n}-v_{j-1}^{n}\right)+H_{j}^{n} \tilde{e}_{j}^{n},
\end{gathered}
$$

where

$$
\begin{aligned}
E_{j}^{n} & =A_{j}^{n}+D_{j}^{n}\left(I-D_{j}^{n}\right)^{-1} A_{j}^{n}, \\
F_{j}^{n} & =B_{j}^{n}+\frac{1}{2} D_{j}^{n}\left(I-D_{j}^{n}\right)^{-1}\left(v-\lambda \Lambda_{j+1}^{n}+2 B_{j}^{n}\right), \\
G_{j}^{n} & =C_{j}^{n}-\frac{1}{2} D_{j}^{n}\left(I-D_{j}^{n}\right)^{-1}\left(v+\lambda \Lambda_{j}^{n}-2 C_{j}^{n}\right), \\
H_{j}^{n} & =I+D_{j}^{n}\left(I-D_{j}^{n}\right)^{-1} .
\end{aligned}
$$

In the rest of this section, abusing notation slightly we denote by $|A|$ the matrix (vector) whose components are the absolute values of the corresponding components of a matrix (vector) $A$, and by $\operatorname{diag}(A)$ the diagonal matrix consisting of the diagonal elements of matrix $A$, i.e.,

$$
|A|=\left(\left|a_{i, j}\right|\right), \quad \operatorname{diag}(A)=\operatorname{diag}\left(a_{11}, \ldots, a_{m m}\right) \text { for } A=\left(a_{i, j}\right) .
$$

We now rewrite (4.3) as

$$
\begin{aligned}
& v_{j}^{n+1}-\frac{1}{2}\left(v+\lambda \Lambda_{j}^{n}-2 \operatorname{diag} G_{j}^{n}\right) v_{j-1}^{n}-\frac{1}{2}\left(v-\lambda \Lambda_{j+1}^{n}+2 \operatorname{diag} F_{j}^{n}\right) v_{j+1}^{n} \\
& \quad-\left(1-v+\frac{\lambda}{2}\left(\Lambda_{j+1}^{n}-\Lambda_{j}^{n}\right)-\operatorname{diag}\left(F_{j}^{n}-G_{j}^{n}\right)\right) v_{j}^{n} \\
& =E_{j}^{n} v_{j}^{n}+\left(F_{j}^{n}-\operatorname{diag} F_{j}^{n}\right)\left(v_{j+1}^{n}-v_{j}^{n}\right)+\left(G_{j}^{n}-\operatorname{diag} G_{j}^{n}\right)\left(v_{j}^{n}-v_{j-1}^{n}\right)+H_{j}^{n} \tilde{e}_{j}^{n} .
\end{aligned}
$$

By the definition of the matrices $A_{j}^{n}, \ldots, G_{j}^{n}$, each component in these matrices has a bound of order $O(1) \varepsilon$ due to Corollary 2.1; consequently, all the matrices on the left-hand side of (4.6) are diagonal and positive for small $\varepsilon$ and $\lambda$. This immediately implies that

$$
\begin{aligned}
\left|v_{j}^{n+1}\right|-\frac{1}{2}( & \left.v+\lambda \Lambda_{j}^{n}-2 \operatorname{diag} G_{j}^{n}\right)\left|v_{j-1}^{n}\right|-\frac{1}{2}\left(v-\lambda \Lambda_{j+1}^{n}+2 \operatorname{diag} F_{j}^{n}\right)\left|v_{j+1}^{n}\right| \\
& -\left(1-v+\frac{\lambda}{2}\left(\Lambda_{j+1}^{n}-\Lambda_{j}^{n}\right)-\operatorname{diag}\left(F_{j}^{n}-G_{j}^{n}\right)\right)\left|v_{j}^{n}\right| \\
\leqq & \left|E_{j}^{n}\right|\left|v_{j}^{n}\right|+\left|F_{j}^{n}-\operatorname{diag} F_{j}^{n}\right|\left|v_{j+1}^{n}-v_{j}^{n}\right| \\
& +\left|G_{j}^{n}-\operatorname{diag} G_{j}^{n}\right|\left|v_{j}^{n}-v_{j-1}^{n}\right|+\left|H_{j}^{n}\right|\left|\hat{e}_{j}^{n}\right| .
\end{aligned}
$$


The principal part of (4.7) can be rewritten in the conservation form

$$
\begin{aligned}
\left|v_{j}^{n+1}\right|-\left|v_{j}^{n}\right| & +\frac{\lambda}{2} \Lambda_{j+1}^{n}\left(\left|v_{j+1}^{n}\right|-\left|v_{j}^{n}\right|\right)+\frac{\lambda}{2} \Lambda_{j}^{n}\left(\left|v_{j}^{n}\right|-\left|v_{j-1}^{n}\right|\right) \\
& -\frac{v}{2}\left(\left|v_{j+1}^{n}\right|-2\left|v_{j}^{n}\right|+\left|v_{j-1}^{n}\right|\right) \\
& -\operatorname{diag} F_{j}^{n}\left(\left|v_{j+1}^{n}\right|-\left|v_{j}^{n}\right|\right)+\operatorname{diag} G_{j}^{n}\left(\left|v_{j}^{n}\right|-\left|v_{j-1}^{n}\right|\right) \\
\leqq & \left|E_{j}^{n}\right|\left|v_{j}^{n}\right|+\left|F_{j}^{n}-\operatorname{diag} F_{j}^{n}\right|\left(\left|v_{j+1}^{n}\right|+\left|v_{j}^{n}\right|\right) \\
& +\left|G_{j}^{n}-\operatorname{diag} G_{j}^{n}\right|\left(\left|v_{j}^{n}\right|+\left|v_{j-1}^{n}\right|\right)+\left|H_{j}^{n}\right|\left|\tilde{e}_{j}^{n}\right| .
\end{aligned}
$$

Here and in what follows, the vector inequality is to be understood componentwise. Summing (4.8) by parts over $j$ we obtain

$$
\sum_{j}\left(\left|v_{j}^{n+1}\right|-\left|v_{j}^{n}\right|\right)+\lambda \sum_{j}\left(\Lambda_{j}^{n}-\Lambda_{j+1}^{n}\right)\left|v_{j}^{n}\right| \leqq I_{j}^{n}\left|v_{j}^{n}\right|+\left|H_{j}^{n}\right|\left|\tilde{e}_{j}^{n}\right|
$$

where

$$
\begin{aligned}
I_{j}^{n}= & \operatorname{diag}\left(F_{j-1}^{n}-F_{j}^{n}\right)+\operatorname{diag}\left(G_{j+1}^{n}-G_{j}^{n}\right)+\left|E_{j}^{n}\right|+\left|F_{j-1}^{n}-\operatorname{diag} F_{j-1}^{n}\right| \\
& +\left|F_{j}^{n}-\operatorname{diag} F_{j}^{n}\right|+\left|G_{j}^{n}-\operatorname{diag} G_{j}^{n}\right|+\left|G_{j+1}^{n}-\operatorname{diag} G_{j+1}^{n}\right| .
\end{aligned}
$$

In order to control waves in transversal directions, we multiply both sides of (4.9) by a constant diagonal matrix $W=\operatorname{diag}\left(w_{1}, \ldots, w_{m}\right)$ with $w_{k}=1$, to obtain

$$
\begin{aligned}
\sum_{j}\left(\left|W v_{j}^{n+1}\right|-\left|W v_{j}^{n}\right|\right)+\lambda \sum_{j} W\left(\Lambda_{j}^{n}-\Lambda_{j+1}^{n}\right)\left|v_{j}^{n}\right| \\
\quad \leqq \sum_{j} W I_{j}^{n}\left|v_{j}^{n}\right|+\sum_{j} W\left|H_{j}^{n}\right|\left|\tilde{e}_{j}^{n}\right| .
\end{aligned}
$$

We now estimate each term on the right-hand side of (4.11). From (4.2), (4.4) and Corollary 2.1 it follows that

$$
\begin{gathered}
\operatorname{diag}\left(F_{j-1}^{n}-F_{j}^{n}\right)+\operatorname{diag}\left(G_{j+1}^{n}-G_{j}^{n}\right)=O(\varepsilon)\left(\lambda_{k, j}^{n}-\lambda_{k, j+1}^{n}\right), \\
E_{j}^{n}=\left(L_{j}^{n+1}-L_{j}^{n}+\frac{\lambda}{2} \Lambda_{j}^{n}\left(L_{j+1}^{n}-L_{j-1}^{n}\right)\right) R_{j}^{n}+O(\varepsilon)\left(\lambda_{k, j}^{n}-\lambda_{k, j+1}^{n}\right) .
\end{gathered}
$$

Following the argument in (3.14) and (3.15), we can show that the $(k, k)$-th element of the matrix

$$
L_{j}^{n+1}-L_{j}^{n}+\frac{\lambda}{2} \Lambda_{j}^{n}\left(L_{j+1}^{n}-L_{j-1}^{n}\right)
$$

is of order $O(\varepsilon)\left(\lambda_{k, j}^{n}-\lambda_{k, j+1}^{n}\right)$ and the remaining elements are of order $O(1)\left(\lambda_{k, j}^{n}-\lambda_{k, j+1}^{n}\right)$. Therefore,

$$
|W| E_{j}^{n}|| v_{j}^{n}|| \leqq O(1)\left(\lambda_{k, j}^{n}-\lambda_{k, j+1}^{n}\right)\left(\left(\varepsilon+\sum_{\mu \neq k} w_{\mu}\right)\left|v_{k, j}^{n}\right|+\sum_{\mu \neq k}\left|v_{\mu, j}^{n}\right|\right) .
$$


As a consequence of (4.11), (4.12) and the fact that the diagonal elements of the matrix

$$
\left|F_{j-1}^{n}-\operatorname{diag} F_{j-1}^{n}\right|+\left|F_{j}^{n}-\operatorname{diag} F_{j}^{n}\right|+\left|G_{j}^{n}-\operatorname{diag} G_{j}^{n}\right|+\left|G_{j+1}^{n}-\operatorname{diag} G_{j+1}^{n}\right|
$$

are zero and the remaining elements of the matrix are of order $O(1)\left(\lambda_{k, j}^{n}-\right.$ $\left.\lambda_{k, j+1}^{n}\right)$, we have

$$
|W| I_{j}^{n}|| v_{j}^{n}|| \leqq O(1)\left(\lambda_{k, j}^{n}-\lambda_{k, j+1}^{n}\right)\left(\left(\varepsilon+\sum_{\mu \neq k} w_{\mu}\right)\left|v_{k, j}^{n}\right|+\sum_{\mu \neq k}\left|v_{\mu, j}^{n}\right|\right) .
$$

Direct computation using Corollary 2.1 shows that

$$
\left|\tilde{e}_{j}^{n}\right| \leqq O(\varepsilon)\left(\lambda_{k, j}^{n}-\lambda_{k, j+1}^{n}\right)\left|v_{j}^{n}\right|+O(1)\left|u_{j}^{n}-\phi_{j}^{n}\right|^{2} .
$$

Therefore,

$$
\begin{aligned}
& \sum_{j}\left(\left|W v_{j}^{n+1}\right|-\left|W v_{j}^{n}\right|\right)+\lambda \sum_{j} W\left(\Lambda_{j}^{n}-\Lambda_{j+1}^{n}\right)\left|v_{j}^{n}\right| \\
& \leqq O(1) \sum_{j}\left(\lambda_{k, j}^{n}-\lambda_{k, j+1}^{n}\right)\left(\left(\varepsilon+\sum_{\mu \neq k} w_{\mu}\right)\left|v_{k, j}^{n}\right|+\sum_{\mu \neq k}\left|v_{\mu, j}^{n}\right|\right)+O(1) \sum_{j}\left|u_{j}^{n}-\phi_{j}^{n}\right|^{2} .
\end{aligned}
$$

For suitably small $\varepsilon, \omega_{\mu} \mu \neq k$, we have

$$
\begin{aligned}
\sum_{j}\left(\left|W v_{j}^{n+1}\right|\right. & \left.-\left|W v_{j}^{n}\right|\right)+\frac{\lambda}{2} \sum_{j}\left(\lambda_{k, j}^{n}-\lambda_{k, j+1}^{n}\right)\left|v_{k, j}^{n}\right| \\
& \leqq O(1) \sum_{j}\left(\lambda_{k, j}^{n}-\lambda_{k, j+1}^{n}\right) \sum_{\mu \neq k}\left|v_{\mu, j}^{n}\right|+O(1)\left|u_{j}^{n}-\phi_{j}^{n}\right|^{2} .
\end{aligned}
$$

The first term on the right-hand side of (4.15) is estimated by the following proposition which will be proved at the end of this section.

Proposition 4.1 (Estimate on the transversal waves). For suitably small $\varepsilon$ and $\lambda$ the following estimate holds:

$$
\begin{aligned}
& \sum_{j, n \leqq n_{2}}\left(\lambda_{k, j}^{n}-\lambda_{k, j+1}^{n}\right) \sum_{\mu \neq k}\left|v_{\mu, j}^{n}\right| \\
& \quad \leqq O(\varepsilon) \sum_{j}\left(\left|v_{j}^{0}\right|+\left|v_{j}^{0}\right|^{2}\right)+O(\varepsilon) \sum_{j, n \leqq n_{2}}\left(\lambda_{k, j}^{n}-\lambda_{k, j+1}^{n}\right)\left|v_{k, j}^{n}\right| .
\end{aligned}
$$

Assuming this proposition, we have from (4.15) and (4.16) that

$$
\begin{aligned}
\sum_{j}\left|v_{j}^{n_{2}}\right|+\sum_{j, n \leqq n_{2}}\left(\lambda_{k, j}^{n}-\lambda_{k, j+1}^{n}\right)\left|v_{k, j}^{n}\right| & \\
& \leqq O(1) \sum_{j}\left(\left|v_{j}^{0}\right|+\left|v_{j}^{0}\right|^{2}\right)+O(1) \sum_{j, n \leqq n_{2}}\left|u_{j}^{n}-\phi_{j}^{n}\right|^{2} .
\end{aligned}
$$


But Proposition 3.2 shows that the last term on the right-hand side of (4.17) is bounded above by $O(1) M(0)^{2}$. Thus we have shown

Proposition 4.2. Assume that $\varepsilon$ and $M(0)$ are suitably small. Then the problem (4.1) has a unique global solution $v_{j}^{n}$ satisfying

$$
\sup _{n} \sum_{j}\left|v_{j}^{n}\right|+\sum_{j, n}\left|\lambda_{k, j}^{n}-\lambda_{k, j+1}^{n}\right|\left|v_{j}^{n}\right| \leqq O(1) \sum_{j}\left(\left|v_{j}^{0}\right|+\left|v_{j}^{0}\right|^{2}\right) \quad \text { for any } n \geqq 0 \text {. }
$$

This immediately yields the desired $L^{1}$-stability estimate.

Proof of the Second Part of Theorem 1.1. As in the proof of the first part of Theorem 1.1, we can show that $(1.7 \mathrm{a})$ and $(1.7 \mathrm{~d})$ imply that

$$
\sum_{j}\left|\bar{v}_{j}^{0}\right|<\infty \text {. }
$$

Thus Proposition 4.2 and (3.1) show that

$$
\sum_{j}\left|u_{j}^{n}-\phi_{j}^{n}\right| \leqq 2 \sum_{j}\left|\bar{v}_{j}^{n}\right|<\infty,
$$

which yields the desired estimate (1.9). This completes the proof of Theorem 1.1.

It remains to derive the estimate (4.16) on the transversal waves. As in the proof of Proposition 3.1, we employ a modified version of the "vertical estimate". However, in the $L^{1}$-estimate, the dissipation terms do not produce terms with a favorable sign, in contrast to the previous case (see (4.15)); additional care is needed to relate a sum along a time-like grid segment to a sum along a space grid segment (see the proof of (4.22)).

Proof of Proposition 4.1. Fix $\mu>k$ and assume that $p \geqq 0$ (the case $p \leqq 0$ can be treated similarly). Let $N$ be any given positive integer, $j_{0}$ a given space index, and $J\left(j_{0}\right)$ the time-like grid line originating at $\left(j_{0}, 0\right)$ and defined by (3.25). For any integer $n \in[0, N q-1]$, summing the $\mu$-th inequality in (4.8) by parts over $j$ from $-\infty$ to $j_{n}$, and using known estimates we obtain

$$
\begin{aligned}
& \sum_{j=-\infty}^{j_{n}}\left(\left|v_{\mu, j}^{n+1}\right|-\left|v_{\mu, j}^{n}\right|\right)+\frac{p}{q}\left|v_{\mu, j_{n}}^{n}\right|+\lambda\left(\lambda_{\mu, j_{n}}^{n}-s\right)\left|v_{\mu, j_{n}}^{n}\right| \\
& \leqq \sum_{j=-\infty}^{j_{n}}\left(I_{j}^{n}\left|v_{j}^{n}\right|\right)_{\mu}+\frac{1}{2}\left(v-\lambda \lambda_{\mu, j_{n}+1}^{n}\right)\left(\left|v_{\mu, j_{n}+1}^{n}\right|-\left|v_{\mu, j_{n}}^{n}\right|\right) \\
&+\left(\lambda_{\mu, j_{n}}^{n}-\lambda_{\mu, j_{n}+1}^{n}\right)\left|v_{\mu, j_{n}}^{n}\right|+\left(\left(\operatorname{diag} F_{j_{n}}^{n}+\left|F_{j_{n}}^{n}-\operatorname{diag} F_{j_{n}}^{n}\right|\right)\left|v_{j_{n}+1}^{n}\right|\right)_{\mu} \\
&-\left(\left(\operatorname{diag} G_{j_{n}+1}^{n}+\left|G_{j_{n}+1}^{n}-\operatorname{diag} G_{j_{n}+1}^{n}\right|\right)\left|v_{j_{n}}^{n}\right|\right)_{\mu}+\sum_{j=-\infty}^{j_{n}}\left(\left|H_{j}^{n}\right|\left|\tilde{e}_{j}^{n}\right|\right)_{\mu} \\
& \leqq O(1) \sum_{j}\left(\lambda_{k, j}^{n}-\lambda_{k, j+1}^{n}\right)\left|v_{j}^{n}\right|+O(1) \sum_{j}\left|u_{j}^{n}-\phi_{j}^{n}\right|^{2} \\
&+\frac{1}{2}\left(v-\lambda \lambda_{\mu, j_{n}+1}^{n}\right)\left(\left|v_{\mu, j_{n}+1}^{n}\right|-\left|v_{\mu, j_{n}}^{n}\right|\right) .
\end{aligned}
$$


Summing this inequality over $n$ from 0 to $N q-1$ gives

$$
\begin{aligned}
\sum_{n=0}^{N q-1} \sum_{j=-\infty}^{j_{n}}\left(\left|v_{\mu, j}^{n+1}\right|\right. & \left.-\left|v_{\mu, j}^{n}\right|\right)+\frac{p}{q} \sum_{n=0}^{N q-1}\left|v_{\mu, j_{n}}^{n}\right|+\lambda \sum_{n=0}^{N q-1}\left(\lambda_{\mu, j_{n}}^{n}-s\right)\left|v_{\mu, j_{n}}^{n}\right| \\
\leqq & O(1) \sum_{n=0}^{N q-1} \sum_{j=-\infty}^{\infty}\left|\lambda_{k, j}^{n}-\lambda_{k, j+1}^{n}\right|\left|v_{j}^{n}\right|+O(1) \sum_{n=0}^{N q-1} \sum_{j=-\infty}^{\infty}\left|u_{j}^{n}-\phi_{j}^{n}\right|^{2} \\
& +\frac{1}{2} \sum_{n=0}^{N q-1}\left(v-\lambda \lambda_{\mu, j_{n}+1}^{n}\right)\left(\left|v_{\mu, j_{n}+1}^{n}\right|-\left|v_{\mu, j_{n}}^{n}\right|\right)
\end{aligned}
$$

Since

$$
\begin{aligned}
\sum_{n=0}^{N q-1} & \sum_{j=-\infty}^{j_{n}}\left(\left|v_{\mu, j}^{n+1}\right|-\left|v_{\mu, j}^{n}\right|\right)+\frac{p}{q} \sum_{n=0}^{N q-1}\left|v_{\mu, j_{n}}^{n}\right| \\
& =\sum_{j=-\infty}^{j_{0}+N q}\left|v_{\mu, j}^{N q}\right|-\sum_{j=-\infty}^{j_{0}-p}\left|v_{\mu, j}^{0}\right|+\frac{1}{q} \sum_{n=0}^{N-1}\left(p \sum_{\alpha=0}^{q-1}\left|v_{\mu, j_{n q}}^{n q+\alpha}\right|-q \sum_{\beta=-p+1}^{0}\left|v_{\mu, j_{n q}+\beta}^{n q}\right|\right)
\end{aligned}
$$

it follows from (4.19) that

$$
\begin{aligned}
& \lambda \sum_{n=0}^{N q-1}\left(\lambda_{\mu, j_{n}}^{n}-s\right)\left|v_{\mu, j_{n}}^{n}\right| \\
& \quad \leqq \sum_{j}\left|v_{\mu, j}^{0}\right|+O(1) \sum_{n=0}^{N q-1} \sum_{j=-\infty}^{\infty}\left|\lambda_{k, j}^{n}-\lambda_{k, j+1}^{n}\right|\left|v_{j}^{n}\right| \\
& \quad+O(1) \sum_{n=0}^{N q-1} \sum_{j=-\infty}^{\infty}\left|u_{j}^{n}-\phi_{j}^{n}\right|^{2}+\frac{1}{2} \sum_{n=0}^{N q-1}\left(v-\lambda \lambda_{\mu, j_{n}+1}^{n}\right)\left(\left|v_{\mu, j_{n}+1}^{n}\right|-\left|v_{\mu, j_{n}}^{n}\right|\right) \\
& \quad-\frac{1}{q} \sum_{n=0}^{N-1}\left(p \sum_{\alpha=0}^{q-1}\left|v_{\mu, j_{n q}}^{n q+\alpha}\right|-q \sum_{\beta=-p+1}^{0}\left|v_{\mu, j_{n q}+\beta}^{n q}\right|\right) .
\end{aligned}
$$

We now multiply both sides of (4.20) by $\lambda_{k, j_{0}}^{0}-\lambda_{k, j_{0}+1}^{0}$ and sum over $j_{0}$ from $-\infty$ to $\infty$ to obtain

$$
\begin{aligned}
& \lambda \sum_{j_{0}=-\infty}^{\infty}\left(\lambda_{k, j_{0}}^{0}-\lambda_{k, j_{0}+1}^{0}\right) \sum_{n=0}^{N q-1}\left(\lambda_{\mu, j_{n}}^{n}-s\right)\left|v_{\mu, j_{n}}^{n}\right| \\
& \leqq \\
& O(\varepsilon) \sum_{j}\left(\left|v_{j}^{0}\right|+\left|v_{j}^{0}\right|^{2}\right)+O(\varepsilon) \sum_{n=0}^{N q-1} \sum_{j=-\infty}^{\infty}\left|\lambda_{k, j}^{n}-\lambda_{k, j+1}^{n}\right|\left|v_{j}^{n}\right| \\
& \quad+\frac{1}{2} \sum_{j_{0}=-\infty}^{\infty}\left(\lambda_{k, j_{0}}^{0}-\lambda_{k, j_{0}+1}^{0}\right) \sum_{n=0}^{N q-1}\left(v-\lambda \lambda_{\mu, j_{n}+1}^{n}\right)\left(\left|v_{\mu, j_{n}+1}^{n}\right|-\left|v_{\mu, j_{n}}^{n}\right|\right) \\
& \quad-\frac{1}{q} \sum_{n=0}^{N-1} \sum_{j_{0}=-\infty}^{\infty}\left(\lambda_{k, j_{0}}^{0}-\lambda_{k, j_{0}+1}^{0}\right)\left(p \sum_{\alpha=0}^{q-1}\left|v_{\mu, j_{n q}}^{n q+\alpha}\right|-q \sum_{\beta=-p+1}^{0}\left|v_{\mu, j_{n q}+\beta}^{n q}\right|\right),
\end{aligned}
$$


where the $L^{2}$-estimate (3.23) has been used. We now estimate the last two terms on the right-hand side of (4.21). First we assert that

$$
\begin{aligned}
& \sum_{n=0}^{N-1} \sum_{j_{0}=-\infty}^{\infty}\left(\lambda_{k, j_{0}}^{0}-\lambda_{k, j_{0}+1}^{0}\right)\left(p \sum_{\alpha=0}^{q-1}\left|v_{\mu, j_{n q}}^{n q+\alpha}\right|-q \sum_{\beta=-p+1}^{0}\left|v_{\mu, j_{n q}+\beta}^{n q}\right|\right) \\
& \leqq O(\varepsilon) \sum_{n=0}^{N-1} \sum_{j}\left(\lambda_{k, j}^{n}-\lambda_{k, j+1}^{n}\right)\left|v_{j}^{n}\right|+O(\varepsilon) \sum_{j, n}\left|u_{j}^{n}-\phi_{j}^{n}\right|^{2} .
\end{aligned}
$$

Next, summation by parts shows that

$$
\begin{aligned}
\frac{1}{2} \sum_{j_{0}=-\infty}^{\infty}\left(\lambda_{k, j_{0}}^{0}-\lambda_{k, j_{0}+1}^{0}\right) \sum_{n=0}^{N q-1}\left(v-\lambda \lambda_{\mu, j_{n}+1}^{n}\right)\left(\left|v_{\mu, j_{n}+1}^{n}\right|-\left|v_{\mu, j_{n}}^{n}\right|\right) \\
\leqq O(\varepsilon) \sum_{j_{0}=-\infty}^{\infty}\left(\lambda_{k, j_{0}}^{0}-\lambda_{k, j_{0}+1}^{0}\right) \sum_{n=0}^{N q-1}\left(\lambda_{m u, j_{n}}^{n}-s\right)\left|v_{\mu, j_{n}}^{n}\right|
\end{aligned}
$$

In fact,

$$
\begin{aligned}
\sum_{j_{0}=-\infty}^{\infty} & \left(\lambda_{k, j_{0}}^{0}-\lambda_{k, j_{0}+1}^{0}\right) \sum_{n=0}^{N q-1}\left(v-\lambda \lambda_{\mu, j_{n}+1}^{n}\right)\left(\left|v_{\mu, j_{n}+1}^{n}\right|-\left|v_{\mu, j_{n}}^{n}\right|\right) \\
= & \sum_{n=0}^{N-1} \sum_{\alpha=0}^{q-1} \sum_{j_{n}=-\infty}^{\infty}\left(\lambda_{k, j_{n q}}^{n q}-\lambda_{k, j_{n q}+1}^{n q}\right)\left(v-\lambda \lambda_{\mu, j_{n q}+1}^{n q+\alpha}\right)\left(\left|v_{\mu, j_{n q}+1}^{n q+\alpha}\right|-\left|v_{\mu, j_{n q}}^{n q+\alpha}\right|\right) \\
= & -\sum_{n=0}^{N-1} \sum_{\alpha=0}^{q-1} \sum_{j_{n}=-\infty}^{\infty}\left(\left(\lambda_{k, j_{n q}}^{n q}-\lambda_{k, j_{n q}+1}^{n q}\right)\left(v-\lambda \lambda_{\mu, j_{n q}+1}^{n q+\alpha+\alpha}\right)\right. \\
& \left.-\left(\lambda_{k, j_{n q}}^{n q}-1-\lambda_{k, j_{n q}}^{n q}\right)\left(v-\lambda \lambda_{\mu, j_{n q}}^{n q+\alpha}\right)\right)\left|v_{\mu, j_{n q}}^{n q+\alpha}\right| \\
\leqq & O(\varepsilon) \sum_{n=0}^{N-1} \sum_{\alpha=0}^{q-1} \sum_{j_{n}=-\infty}^{\infty}\left(\lambda_{k, j_{n q}}^{n q}-\lambda_{k, j_{n q}+1}^{n q}\right)\left|v_{\mu, j_{n q}}^{n q+\alpha}\right| \\
\leqq & O(\varepsilon) \sum_{j_{0}=-\infty}^{\infty}\left(\lambda_{k, j_{0}}^{0}-\lambda_{k, j_{0}+1}^{0}\right) \sum_{n=0}^{N q-1}\left(\lambda_{k, j_{n}}^{n}-s\right)\left|v_{\mu, j_{n}}^{n}\right| .
\end{aligned}
$$

Assuming for a moment that (4.22a) holds, we find that

$$
\begin{aligned}
& \sum_{j_{0}=-\infty}^{\infty}\left(\lambda_{k, j_{0}}^{0}-\lambda_{k, j_{0}+1}^{0}\right) \sum_{n=0}^{N q-1}\left|v_{\mu, j_{n}}^{n}\right| \\
& \quad \leqq O(\varepsilon) \sum_{j}\left(\left|v_{j}^{0}\right|+\left|v_{j}^{0}\right|^{2}\right)+O(\varepsilon) \sum_{n=0}^{N q-1} \sum_{j=-\infty}^{\infty}\left(\lambda_{k, j}^{n}-\lambda_{k, j+1}^{n}\right)\left|v_{j}^{n}\right| .
\end{aligned}
$$


Noting that

$$
\begin{aligned}
\sum_{n=0}^{N q-1} \sum_{j=-\infty}^{\infty}\left(\lambda_{k, j}^{n}-\lambda_{k, j+1}^{n}\right)\left|v_{\mu, j}^{n}\right| & =\sum_{j_{0}=-\infty}^{\infty} \sum_{n=0}^{N q-1}\left(\lambda_{k, j_{n}}^{n}-\lambda_{k, j_{n}+1}^{n}\right)\left|v_{\mu, j_{n}}^{n}\right| \\
& \leqq(1+O(\varepsilon)) \sum_{j_{0}=-\infty}^{\infty}\left(\lambda_{k, j}^{0}-\lambda_{k, j+1}^{0}\right) \sum_{n=0}^{N q-1}\left|v_{\mu, j_{n}}^{n}\right|,
\end{aligned}
$$

we conclude from (4.23) that

$$
\sum_{j, n}\left(\lambda_{k, j}^{n}-\lambda_{k, j+1}^{n}\right)\left|v_{\mu, j}^{n}\right| \leqq O(\varepsilon) \sum_{j=-\infty}^{\infty}\left(\left|v_{j}^{0}\right|+\left|v_{j}^{0}\right|^{2}\right)+O(\varepsilon) \sum_{j, n}\left(\lambda_{k, j}^{n}-\lambda_{k, j+1}^{n}\right)\left|v_{j}^{n}\right|
$$

Similar analysis shows that (4.24) is also true for $\mu<k$. Therefore, summation over $\mu$ yields

$$
\begin{aligned}
\sum_{j, n}\left(\lambda_{k, j}^{n}-\lambda_{k, j+1}^{n}\right) \sum_{\mu \neq k}\left|v_{\mu, j}^{n}\right| \leqq & O(\varepsilon) \sum_{j}\left(\left|v_{j}^{0}\right|+\left|v_{j}^{0}\right|^{2}\right) \\
& +O(\varepsilon) \sum_{j, n}\left(\lambda_{k, j}^{n}-\lambda_{k, j+1}^{n}\right)\left|v_{j}^{n}\right|,
\end{aligned}
$$

which is the desired estimate. It remains to show that $(4.22 \mathrm{a})$ is true. We first rewrite the left-hand side of (4.22a) as

$$
\begin{aligned}
p \sum_{n=0}^{N-1} & \sum_{\alpha=0}^{q-1} \sum_{j_{0}=-\infty}^{\infty}\left(\lambda_{k, j_{n q}}^{n q}-\lambda_{k, j_{n q}+1}^{n q}\right)\left(\left|v_{\mu, j_{n q}}^{n q+\alpha}\right|-\left|v_{\mu, j_{n q}}^{n q}\right|\right) \\
& -q \sum_{n=0}^{N-1} \sum_{i=1}^{p} \sum_{j_{0}=-\infty}^{\infty}\left(\lambda_{k, j_{n q}}^{n q}-\lambda_{k, j_{n q}+1}^{n q}\right)\left(\left|v_{\mu, j_{n q}+i}^{n q}\right|-\left|v_{\mu, j_{n q}}^{n q}\right|\right) \\
= & p \sum_{n=0}^{N-1} \sum_{\alpha=0}^{q-1} \sum_{l=0}^{\alpha-1} \sum_{j_{0}=-\infty}^{\infty}\left(\lambda_{k, j}^{n q}-\lambda_{k, j+1}^{n q}\right)\left(\left|v_{\mu, j}^{n q+l+1}\right|-\left|v_{\mu, j}^{n q+l}\right|\right) \\
& -q \sum_{n=0}^{N-1} \sum_{i=-p+1}^{0} \sum_{l=0}^{i-1} \sum_{j=-\infty}^{\infty}\left(\lambda_{k, j}^{n q}-\lambda_{k, j+1}^{n q}\right)\left(\left|v_{\mu, j}^{n q}+l+1\right|-\left|v_{\mu, j+l}^{n q}\right|\right) \\
\equiv & I_{1}+I_{2} .
\end{aligned}
$$

But

$$
\begin{aligned}
& \sum_{j=-\infty}^{\infty}\left(\lambda_{k, j}^{n q_{j}}-\lambda_{k, j+1}^{n q}\right)\left(\left|v_{\mu, j+l+1}^{n q}\right|-\left|v_{\mu, j+l}^{n q}\right|\right) \\
& =\sum_{j=-\infty}^{\infty}\left(\lambda_{k, j-l+1}^{n q}-2 \lambda_{k, j-l}^{n q}+\lambda_{k, j-l-1}^{n q}\right)\left|v_{\mu, j}^{n q}\right| \\
& \leqq O(\varepsilon) \sum_{j=-\infty}^{\infty}\left(\lambda_{k, j}^{n q}-\lambda_{k, j+1}^{n q}\right)\left|v_{\mu, j}^{n q}\right|
\end{aligned}
$$


This leads to

$$
\left|I_{1}\right| \leqq O(\varepsilon) \sum_{j, n}\left(\lambda_{k, j}^{n}-\lambda_{k, j+1}^{n}\right)\left|v_{j}^{n}\right|
$$

Using inequality (4.8) and summation by parts, we verify that

$$
\begin{aligned}
\sum_{j=-\infty}^{\infty}\left(\lambda_{k, j}^{n q}-\right. & \left.\lambda_{k, j+1}^{n q}\right)\left(\left|v_{\mu, j}^{n q+l+1}\right|-\left|v_{\mu, j}^{n q+l}\right|\right) \\
\leqq & -\frac{\lambda}{2} \sum_{j=-\infty}^{\infty}\left(\lambda_{k, j}^{n q}-\lambda_{k, j+1}^{n q}\right) \lambda_{\mu, j}^{n q+l}\left(\left|v_{\mu, j+1}^{n q+l}\right|-\left|v_{\mu, j-1}^{n q+l}\right|\right) \\
& -\frac{v}{2} \sum_{j=-\infty}^{\infty}\left(\lambda_{k, j}^{n q}-\lambda_{k, j+1}^{n q}\right) \lambda_{\mu, j}^{n q+l}\left(\left|v_{\mu, j+1}^{n q+l}\right|-2\left|v_{\mu, j}^{n q+l}\right|+\left|v_{\mu, j-1}^{n q+l}\right|\right) \\
& +O(\varepsilon) \sum_{j=-\infty}^{\infty}\left(\lambda_{k, j}^{n q}-\lambda_{k, j+1}^{n q}\right) \lambda_{\mu, j}^{n q+l}\left(\left|v_{j+1}^{n q+l}\right|+\left|v_{j}^{n q+l}\right|+\left|v_{j-1}^{n q+l}\right|\right) \\
& +O(\varepsilon) \sum_{j=-\infty}^{\infty}\left|u_{j}^{n q+l}-\phi_{j}^{n q+l}\right|^{2} . \\
\leqq & O(\varepsilon) \sum_{j=-\infty}^{\infty}\left(\lambda_{k, j}^{n q}-\lambda_{k, j+1}^{n q}\right)\left|v_{j}^{n q+l}\right|+O(\varepsilon) \sum_{j=-\infty}^{\infty}\left|u_{j}^{n q+l}-\phi_{j}^{n q+l}\right|^{2} .
\end{aligned}
$$

This leads to

$$
\left|I_{2}\right| \leqq O(\varepsilon) \sum_{j, n}\left(\lambda_{k, j}^{n}-\lambda_{k, j+1}^{n}\right)\left|v_{j}^{n}\right|+O(\varepsilon) \sum_{j, n}\left|u_{j}^{n}-\phi_{j}^{n}\right|^{2},
$$

which yields the desired inequality (4.22a).

\section{Stability Analysis for Multiple Shocks}

In this section, we carry out the $L^{2}$-asymptotic stability analysis of multiple discrete shocks for the Lax-Friedrichs scheme. The main idea is as follows. Since weak waves propagate essentially with characteristic speeds, it follows that waves produced by interactions of shocks from each family are relatively small. This enables us to adapt the ideas developed in previous sections to obtain the desired stability theorem.

We begin by reformulating the problem. Let $\phi_{j}^{n}$ be the discrete multipleshock given by (1.12), i.e.,

$$
\phi_{j}^{n}=\sum_{\mu=1}^{m} \phi_{\mu, j+\delta_{\mu}}^{n}-\sum_{\mu=1}^{m-1} \bar{u}_{\mu}
$$

where $\phi_{\mu}$ is the discrete single-shock profile (1.5) connecting $u_{\mu-1}$ and $u_{\mu}$ with shock speed $s_{\mu}$ satisfying $\lambda s_{\mu}=p_{\mu} / q_{\mu}$ (for $p_{\mu}, q_{\mu} \in \mathbb{Z}$ and $q_{\mu}$ even and positive), and $\delta_{\mu}$ is an integer denoting the phase shift determined by the initial pertur- 
bation. By renormalizing and renaming variables, if necessary, we can assume without loss of generality that $q_{\mu}=q$ and $\delta_{\mu}=0$ for all $\mu=1, \ldots, m$. An easy computation shows that $\phi_{j}^{n}$, given by (5.1), satisfies the Lax-Friedrichs difference equations (1.5 a) approximately:

$\phi_{j}^{n+1}-\phi_{j}^{n}+\frac{\lambda}{2}\left(f\left(\phi_{j+1}^{n}\right)-f\left(\phi_{j-1}^{n}\right)\right)=\frac{v}{2}\left(\phi_{j+1}^{n}-2 \phi_{j}^{n}+\phi_{j-1}^{n}\right)+\frac{\lambda}{2}\left(\eta_{j+1}^{n}-\eta_{j-1}^{n}\right)$

where the error $\eta$ is given by

$$
\eta_{j}^{n}=f\left(\phi_{j}^{n}\right)-\sum_{\mu=1}^{m} f\left(\phi_{\mu, j}^{n}\right)+\sum_{\mu=1}^{m-1} f\left(\bar{u}_{\mu}\right) .
$$

Let $u_{j}^{n}\left(n \leqq n_{1}<\infty\right)$ be a solution to the Lax-Friedrichs scheme (1.5) with initial data satisfying (1.13). To estimate this solution, as in Section 3, we work with the characteristic variables for a summed error equation. We set

$$
\bar{v}_{j}^{n}=\sum_{j=-\infty}^{j}\left(u_{i}^{n}-\phi_{i}^{n}\right), \quad v_{j}^{n}=L_{j}^{n} \bar{v}_{j}^{n},
$$

where a notation such as $L_{j}^{n}=L\left(\phi_{j}^{n}\right)$ has the same form as in Section 3, but with $\phi_{j}^{n}$ defined by (5.1), unless otherwise stated. It follows from (1.5) and (5.1) - (5.4) that

$$
\begin{aligned}
& v_{j}^{n+1}-v_{j}^{n}+\frac{\lambda}{2} \Lambda_{j+1}^{n}\left(v_{j+1}^{n}-v_{j}^{n}\right)+\frac{\lambda}{2} \Lambda_{j}^{n}\left(v_{j}^{n}-v_{j-1}^{n}\right)-\frac{v}{2}\left(v_{j+1}^{n}-2 v_{j}^{n}+v_{j-1}^{n}\right) \\
& =\left(L_{j}^{n+1}-L_{j}^{n}+\frac{\lambda}{2} \Lambda_{j}^{n}\left(L_{j+1}^{n}-L_{j-1}^{n}\right)\right) R_{j}^{n} v_{j}^{n}+e_{j}^{n}+\frac{\lambda}{2} L_{j}^{n}\left(\eta_{j+1}^{n}+\eta_{j}^{n}\right)
\end{aligned}
$$

where $e_{j}^{n}$ has the same form as in (3.6). Taking the scalar product of (5.5) with $2 v_{j}^{n}$ and summing by parts over both the space and time indices, we obtain

$$
\begin{aligned}
\sum_{j}\left|v_{j}^{n_{2}}\right|^{2} & +\lambda \sum_{j, n \leqq n_{2}} \sum_{k=1}^{m}\left(\lambda_{k}\left(\phi_{j}^{n}\right)-\lambda_{k}\left(\phi_{j+1}^{n}\right)\right)\left(v_{k, j}^{n}\right)^{2}+v \sum_{j, n \leqq n_{2}}\left|v_{j+1}^{n}-v_{j}^{n}\right|^{2} \\
= & \sum_{j}\left|v_{j}^{0}\right|^{2}+\sum_{j, n \leqq n_{2}} 2 v_{j}^{n} \cdot\left(L_{j}^{n+1}-L_{j}^{n}+\frac{\lambda}{2} \Lambda_{j}^{n}\left(L_{j+1}^{n}-L_{j-1}^{n}\right)\right) R_{j}^{n} v_{j}^{n} \\
& +\sum_{j, n \leqq n_{2}}\left(2 v_{j}^{n} \cdot e_{j}^{n}+\lambda v_{j}^{n} \cdot L_{j}^{n}\left(\eta_{j+1}^{n}+\eta_{j}^{n}\right)+\left|v_{j}^{n+1}-v_{j}^{n}\right|^{2}\right)
\end{aligned}
$$

We now estimate each term in (5.6) separately. The results are stated in the following five lemmas and a proposition, whose proofs will be given in detail later. In what follows, we assume a priori that $M\left(n_{1}\right)$, defined in (3.13), is bounded, and that $\varepsilon$ is suitably small. 
Lemma 5.1. Let $e_{j}^{n}$ be given by (3.6). Then

$$
\begin{aligned}
\sum_{j, n \leqq n_{2}} 2 v_{j}^{n} \cdot e_{j}^{n} \leqq & \frac{\lambda}{8} \sum_{j, n \leqq n_{2}}\left(\lambda_{k}\left(\phi_{k, j}^{n}\right)-\lambda_{k}\left(\phi_{k, j+1}^{n}\right)\right)\left|v_{j}^{n}\right|^{2} \\
& +O(1)\left(\varepsilon+M\left(n_{1}\right)\right) \sum_{j, n \leqq n_{2}}\left|v_{j+1}^{n}-v_{j}^{n}\right|^{2}+O(\varepsilon) \sum_{j, n \leqq n_{2}}\left|v_{j}^{n+1}-v_{j}^{n}\right|^{2} .
\end{aligned}
$$

Lemma 5.2. The error $\eta_{j}^{n}$ in (5.3), due to the linear superposition of single shocks from each family, is in $L^{1}$ in both space and time; more precisely,

which implies that

$$
\left|\eta_{j}^{n}\right| \leqq O(1) \varepsilon^{2} e^{-O(1) \varepsilon(|j|+n)}
$$

$$
\sum_{j, n \leqq n_{2}}\left|v_{j}^{n} \cdot L_{j}^{n}\left(\eta_{j+1}^{n}+\eta_{j}^{n}\right)\right| \leqq \varepsilon^{1 / 4} \sum_{j, n \leqq n_{2}}\left|v_{j}^{n}-v_{j+1}^{n}\right|^{2}+O(1) \varepsilon^{1 / 4} .
$$

Lemma 5.3. The time difference admits the bound

$$
\begin{aligned}
\sum_{j, n \leqq n_{2}}\left|v_{j}^{n+1}-v_{j}^{n}\right|^{2} \leqq & \left(\left(\lambda|\Lambda|_{L^{\infty}}+v\right)^{2}+O\left(\varepsilon^{1 / 4}\right)+O(1) M\left(n_{1}\right)\right) \sum_{j, n \leqq n_{2}}\left|v_{j+1}^{n}-v_{j}^{n}\right|^{2} \\
& +\frac{\lambda}{8} \sum_{j, n \leqq n_{2}} \sum_{k=1}^{m}\left(\lambda_{k}\left(\phi_{k, j}^{n}\right)-\lambda_{k}\left(\phi_{k, j+1}^{n}\right)\right)\left|v_{j}^{n}\right|^{2}+O(1) \varepsilon^{1 / 4} .
\end{aligned}
$$

Lemma 5.4. The principal waves can be estimated by

$$
\begin{gathered}
\sum_{j, n \leqq n_{2}} \sum_{k=1}^{m}\left(\lambda_{k}\left(\phi_{j}^{n}\right)-\lambda_{k}\left(\phi_{j+1}^{n}\right)\right)\left(v_{k, j}^{n}\right)^{2} \geqq \sum_{j, n \leqq n_{2}} \sum_{k=1}^{m}\left(\lambda_{k}\left(\phi_{k, j}^{n}\right)-\lambda_{k}\left(\phi_{k, j+1}^{n}\right)\right)\left(v_{k, j}^{n}\right)^{2} \\
-O(1) \sum_{j, n \leqq n_{2}} \sum_{k=1}^{m}\left(\lambda_{k}\left(\phi_{k, j}^{n}\right)-\lambda_{k}\left(\phi_{k, j+1}^{n}\right)\right) \sum_{\mu \neq k}\left(v_{\mu, j}^{n}\right)^{2} \\
-\varepsilon^{1 / 4} \sum_{j, n \leqq n_{2}}\left|v_{j}^{n}-v_{j+1}^{n}\right|^{2}-O(1) \varepsilon^{1 / 4}
\end{gathered}
$$

\section{Lemma 5.5.}

$$
\begin{aligned}
& \sum_{j, n \leqq n_{2}} v_{j}^{n} \cdot\left(L_{j}^{n+1}-L_{j}^{n}+\frac{\lambda}{2} \Lambda_{j}^{n}\left(L_{j+1}^{n}-L_{j-1}^{n}\right)\right) R_{j}^{n} v_{j}^{n} \\
& \quad \leqq \frac{\lambda}{8} \sum_{j, n \leqq n_{2}} \sum_{k=1}^{m}\left(\lambda_{k}\left(\phi_{k, j}^{n}\right)-\lambda_{k}\left(\phi_{k, j+1}^{n}\right)\right)\left(v_{k, j}^{n}\right)^{2} \\
& \quad+O(1) \lambda \sum_{j, n \leqq n_{2}} \sum_{k=1}^{m}\left(\lambda_{k}\left(\phi_{k, j}^{n}\right)-\lambda_{k}\left(\phi_{k, j+1}^{n}\right)\right) \sum_{\mu \neq k}\left(v_{\mu, j}^{n}\right)^{2} .
\end{aligned}
$$


Proposition 5.1. The transversal waves can be estimated by

$$
\begin{aligned}
& \sum_{j, n \leqq n_{2}} \sum_{k=1}^{m}\left(\lambda_{k}\left(\phi_{k, j}^{n}\right)-\lambda_{k}\left(\phi_{k, j+1}^{n}\right)\right) \sum_{\mu=k}\left(v_{\mu, j}^{n}\right)^{2} \\
& \leqq O(\varepsilon) \sum_{j}\left|v_{j}^{0}\right|^{2}+O(\varepsilon) \sum_{j, n \leqq n_{2}} \sum_{k=1}^{m}\left(\lambda_{k}\left(\phi_{k, j}^{n}\right)-\lambda_{k}\left(\phi_{k, j+1}^{n}\right)\right)\left(v_{k, j}^{n}\right)^{2} \\
& \quad+O(\varepsilon) \sum_{j, n \leqq n_{2}}\left|v_{j+1}^{n}-v_{j}^{n}\right|^{2}+O(1) \varepsilon^{4 / 3} .
\end{aligned}
$$

Assuming that Lemmas 5.1-5.5 and Proposition 5.1 hold, we continue our energy estimate. It follows from Lemmas 5.1-5.3 that

$$
\begin{aligned}
& \sum_{j, n \leqq n_{2}}\left(2 v_{j}^{n} \cdot e_{j}^{n}+\lambda v_{j}^{n} \cdot L_{j}^{n}\left(\eta_{j+1}^{n}+\eta_{j}^{n}\right)+\left|v_{j}^{n+1}-v_{j}^{n}\right|^{2}\right) \\
& \leqq \frac{\lambda}{4} \sum_{j, n \leqq n_{2}}\left(\lambda_{k}\left(\phi_{k, j}^{n}\right)-\lambda_{k}\left(\phi_{k, j+1}^{n}\right)\right)\left|v_{1}^{n}\right|^{2} \\
& \quad+\left(\left(\lambda|\Lambda|_{L^{\infty}}+v\right)^{2}+O\left(\varepsilon^{1 / 4}\right)+O(1) M\left(n_{1}\right) \sum_{j}\left|v_{j+1}^{n}-v_{j}^{n}\right|^{2}+O(1) \varepsilon^{1 / 4} .\right.
\end{aligned}
$$

This, together with Lemmas 5.4 and 5.5, leads to

$$
\begin{aligned}
& \sum_{j}\left|v_{j}^{n_{2}}\right|^{2}+\frac{\lambda}{2} \sum_{j, n \leqq n_{2}} \sum_{k=1}^{m}\left(\lambda_{k}\left(\phi_{k, j}^{n}\right)-\lambda_{k}\left(\phi_{k, j+1}^{n}\right)\right)\left(v_{k, j}^{n}\right)^{2} \\
& \quad+\left(v-\left(\lambda|\Lambda|_{L^{\infty}}+v\right)^{2}-O\left(\varepsilon^{1 / 4}\right)-O(1) M\left(n_{1}\right)\right) \sum_{j, n \leqq n_{2}}\left|v_{j+1}^{n}-v_{j}^{n}\right|^{2} \\
& \leqq \sum_{j}\left|v_{j}^{0}\right|^{2}+O(1) \lambda \sum_{j, n \leqq n_{2}} \sum_{k=1}^{m}\left(\lambda_{k}\left(\phi_{k, j}^{n}\right)-\lambda_{k}\left(\phi_{k, j+1}^{n}\right)\right) \sum_{\mu \neq k}\left(v_{\mu, j}^{n}\right)^{2}+O(1) \varepsilon^{1 / 4} .
\end{aligned}
$$

Combining this inequality with Proposition 5.1 yields

$$
\begin{aligned}
& \sum_{j}\left|v_{j}^{n_{2}}\right|^{2}+\frac{\lambda}{2} \sum_{j, n \leqq n_{2}} \sum_{k=1}^{m}\left(\lambda_{k}\left(\phi_{k, j}^{n}\right)-\lambda_{k}\left(\phi_{k, j+1}^{n}\right)\right)\left(v_{k, j}^{n}\right)^{2} \\
& \quad+\left(v-\left(\lambda|A|_{L^{\infty}}+v\right)^{2}-O\left(\varepsilon^{1 / 4}\right)-O(1) M\left(n_{1}\right)\right) \sum_{j, n \leqq n_{2}}\left|v_{j+1}^{n}-v_{j}^{n}\right|^{2} \\
& \quad \leqq \sum_{j}\left|v_{j}^{0}\right|^{2}+O(1) \varepsilon^{1 / 4}
\end{aligned}
$$

We thus have demonstrated the following basic a priori estimate. 
Proposition 5.2 (A priori estimate). Let $v_{j}^{n}$ be a solution of (5.6) and (5.7) for $n \leqq n_{1}$. Then there exists a positive constant $c$, independent of $n_{1}$ and $\varepsilon$, such that

$$
\begin{gathered}
\sum_{j}\left|v_{j}^{n_{2}}\right|^{2}+\sum_{j, n \leqq n_{2}}\left|v_{j}^{n}-v_{j+1}^{n}\right|^{2}+\sum_{j, n \leqq n_{2}} \sum_{k=1}^{m}\left(\lambda_{k}\left(\phi_{k, j}^{n}\right)-\lambda_{k}\left(\phi_{k, j+1}^{n}\right)\right)\left(v_{k, j}^{n}\right)^{2} \\
\leqq C\left(M(0)^{2}+\varepsilon^{1 / 4}\right),
\end{gathered}
$$

for all $n_{2} \leqq n_{1}$ provided that $\varepsilon$ and $M\left(n_{1}\right)$ are suitably small.

Having proved the a priori estimate (5.15), we deduce the desired global existence and estimate for the solution to (5.5) just as in Section 3.

Proposition 5.3. Assume that $\varepsilon$ and $M(0)$ are suitably small. Then the problem (5.5) has a unique global solution $v_{j}^{n}$ satisfying, for any $n \geqq 0$,

$$
\begin{gathered}
\sum_{p} \sum_{j}\left|v_{j}^{n}\right|^{2}+\sum_{j, n}\left|v_{j}^{n}-v_{j+1}^{n}\right|^{2}+\sum_{j, n} \sum_{k=1}^{m}\left(\lambda_{k}\left(\phi_{k, j}^{n}\right)-\lambda_{k}\left(\phi_{k, j+1}^{n}\right)\right)\left(v_{k, j}^{n}\right)^{2} \\
\leqq C\left(M^{2}(0)+\varepsilon^{1 / 4}\right)
\end{gathered}
$$

Proof of Theorem 1.2. With Proposition 5.2 at hand, we can prove Theorem 1.2 in the same way as Theorem 1.1. The proof of Theorem 1.2 is considered complete.

The remaining task is to prove Lemmas 5.1-5.5 and Proposition 5.1, which occupies the rest of this section. We begin with Lemma 5.1.

Proof of Lemma 5.1. By direct computation using the expression for $e_{j}^{n}$, (3.6), we can verify that

$$
\begin{aligned}
\sum_{j} v_{j}^{n} \cdot e_{j}^{n} \leqq & O(1) \lambda \sum_{j}\left|\phi_{j}^{n}-\phi_{j+1}^{n}\right|\left|v_{j}^{n}\right|\left|v_{j}^{n+1}-v_{j}^{n}\right| \\
& +O(1) \sum_{j}\left|\phi_{j}^{n}-\phi_{j+1}^{n}\right|\left|v_{j}^{n}\right|\left|v_{j+1}^{n}-v_{j}^{n}\right| \\
& +O(1) \sum_{j}\left|\phi_{j+1}^{n}-2 \phi_{j}^{n}+\phi_{j-1}^{n}\right|\left|v_{j}^{n}\right|^{2}+O(1) M\left(n_{1}\right) \sum_{j}\left|v_{j}^{n}-v_{j-1}^{n}\right|^{2} \\
& +O(\varepsilon) \sum_{j}\left|\phi_{j}^{n}-\phi_{j+1}^{n}\right|\left|v_{j}^{n}\right|^{2}
\end{aligned}
$$

where we have used the facts that

$$
\left|\theta_{j}^{n}\right| \leqq O(1)\left|v_{j}^{n}-v_{j-1}^{n}\right|^{2}+O(1)\left|\phi_{j}^{n}-\phi_{j+1}^{n}\right|^{2}\left|v_{j}^{n}\right|^{2}
$$


and $\left|v_{j}^{n}\right| \leqq M\left(n_{1}\right)$. Since

$$
\begin{gathered}
\left|\phi_{j}^{n}-\phi_{j+1}^{n}\right| \leqq \sum_{k=1}^{m}\left|\phi_{k, j}^{n}-\phi_{k, j+1}^{n}\right| \leqq O(1) \sum_{k=1}^{m}\left(\lambda_{k}\left(\phi_{k, j}^{n}\right)-\lambda\left(\phi_{k, j+1}^{n}\right)\right), \\
\left|\phi_{j+1}^{n}-2 \phi_{j}^{n}-\phi_{j-1}^{n}\right| \leqq O(\varepsilon) \sum_{k=1}^{m}\left(\lambda_{k}\left(\phi_{k, j}^{n}\right)-\lambda\left(\phi_{k, j+1}^{n}\right)\right),
\end{gathered}
$$

as follows from Corollary 2.1, the Cauchy inequality leads to (5.7). This completes the proof of Lemma 5.1.

Proof of Lemma 5.2. First we show (5.8). By the strict hyperbolicity, there are constants $\sigma_{1}, \ldots, \sigma_{m-1}$, such that

$$
-\infty=\sigma_{0}<s_{1}<\sigma_{1}<\cdots<\sigma_{m-1}<s_{m}<\sigma_{m}=\infty .
$$

On the cone $\left\{(j, n): \lambda \sigma_{k-1}<j / n \leqq \lambda \sigma_{k}\right\}$, we rewrite (5.3) as

$$
\begin{aligned}
\eta_{j}^{n}= & f\left(\phi_{k, j}^{n}+\sum_{\mu \neq k} \phi_{\mu, j}^{n}-\sum_{\mu=1}^{m-1} \bar{u}_{\mu}\right)-f\left(\phi_{k, j}^{n}\right) \\
& -\sum_{\mu=1}^{k-1}\left(f\left(\phi_{\mu, j}^{n}\right)-f\left(\bar{u}_{\mu}\right)\right)-\sum_{\mu=k+1}^{m}\left(f\left(\phi_{\mu, j}^{n}\right)-f\left(\bar{u}_{\mu-1}\right)\right) .
\end{aligned}
$$

The Taylor expansion of the right-hand side of (5.17) and Corollary 2.1 lead to

$$
\begin{aligned}
\eta_{j}^{n}= & \nabla f\left(\xi_{k}\right) \sum_{\mu=1}^{k-1}\left(\phi_{\mu, j}^{n}-\bar{u}_{\mu}\right)+\nabla f\left(\xi_{k}\right) \sum_{\mu=k+1}^{m}\left(\phi_{\mu, j}^{n}-\bar{u}_{\mu-1}\right) \\
& -\sum_{\mu=1}^{k-1} \nabla f\left(\xi_{\mu}\right)\left(\phi_{\mu, j}^{n}-\bar{u}_{\mu}\right)-\sum_{\mu=k+1}^{m} \nabla f\left(\xi_{\mu}\right)\left(\phi_{\mu, j}^{n}-\bar{u}_{\mu-1}\right) \\
= & \sum_{\mu=1}^{k-1}\left(\nabla f\left(\xi_{k}\right)-\nabla f\left(\xi_{\mu}\right)\right)\left(\phi_{\mu, j}^{n}-\bar{u}_{\mu}\right)+\sum_{\mu=k+1}^{m}\left(\nabla f\left(\xi_{k}\right)-\nabla f\left(\xi_{\mu}\right)\right)\left(\phi_{\mu, j}^{n}-\bar{u}_{\mu-1}\right) \\
= & O(1) \varepsilon^{2} \sum_{\mu=k} e^{-O(1) \varepsilon\left|j-\lambda s_{\mu} n\right|} .
\end{aligned}
$$

To prove (5.8), we note that for $\mu<k$,

$$
\begin{aligned}
e^{-O(1) \varepsilon\left|j-\lambda s_{\mu} n\right|} & =e^{-O(1) \varepsilon \beta\left|j-\lambda s_{\mu} n\right|} e^{-O(1) \varepsilon(1-\beta)\left|j-\lambda s_{\mu} n\right|} \\
& \leqq e^{-O(1) \varepsilon \beta\left(\sigma_{k-1}-s_{\mu}\right) n} e^{-O(1) \varepsilon(1-\beta)\left(|j|-\lambda\left|s_{\mu}\right| n\right)} \\
& =e^{\left.-O(1) \varepsilon \beta\left(\sigma_{k-1}-s_{\mu}\right)-(1-\beta) \lambda\left|s_{\mu}\right|\right) n} e^{-O(1) \varepsilon \beta|j|} \\
& =e^{-O(1) \varepsilon(|j|+n)}
\end{aligned}
$$

a similar estimate holds for $\mu>k$. Thus the estimate (5.8) follows. 
Next, we prove (5.9). Using the Sobolev and Young inequalities, we have

$$
\begin{aligned}
\sum_{j}\left|v_{j}^{n} \cdot \eta_{j}^{n}\right| & \leqq \sum_{j} O(1) \varepsilon^{2} e^{-O(1) \varepsilon(|j|+n)} \sup _{j}\left|v_{j}^{n}\right|=O(1) \varepsilon e^{-O(1) \varepsilon n} \sup _{j}\left|v_{j}^{n}\right| \\
& \leqq O(1) \varepsilon^{15 / 16} e^{-O(1) \varepsilon n}\left(\sum_{j}\left|v_{j}^{n}-v_{j+1}^{n}\right|^{2}\right)^{1 / 4} \varepsilon^{1 / 16}\left(\sum_{j}\left|v_{j}^{n}\right|^{2}\right)^{1 / 4} \\
& \leqq \varepsilon^{1 / 4} \sum_{j}\left|v_{j}^{n}-v_{j+1}^{n}\right|^{2}+O(1) \varepsilon^{5 / 4} e^{-O(1) \varepsilon n},
\end{aligned}
$$

where we have used the a priori bound on $M\left(n_{1}\right)$. Summing this inequality over $n$ leads to

$$
\sum_{j, n \leqq n_{2}}\left|v_{j}^{n} \cdot \eta_{j}^{n}\right| \leqq \varepsilon^{1 / 4} \sum_{j, n \leqq n_{2}}\left|v_{j}^{n}-v_{j+1}^{n}\right|^{2}+O(1) \varepsilon^{1 / 4},
$$

which yields (5.9).

Proof of Lemma 5.3. It follows from (5.5) and (5.8) that $\left|v_{j}^{n+1}-v_{j}^{n}\right| \leqq \frac{1}{2}\left(\lambda|\Lambda|_{L^{\infty}}+v+O(\varepsilon)+M\left(n_{1}\right)\right)\left(\left|v_{j+1}^{n}-v_{j}^{n}\right|+\left|v_{j}^{n}-v_{j-1}^{n}\right|\right)$

Therefore,

$$
+O(1) \sum_{k=1}^{m}\left(\lambda_{k}\left(\phi_{k, j}^{n}\right)-\lambda_{k}\left(\phi_{k, j+1}^{n}\right)\right)\left|v_{j}^{n}\right|+O(1) \varepsilon^{2} e^{-O(1) \varepsilon(|j|+n)} .
$$

$$
\begin{aligned}
\sum_{j}\left|v_{j}^{n+1}-v_{j}^{n}\right|^{2} \leqq & \left(\lambda|\Lambda|_{L^{\infty}}+v+O(\varepsilon)+M\left(n_{1}\right)\right)^{2} \sum_{j}\left|v_{j+1}^{n}-v_{j}^{n}\right|^{2} \\
& +O(\varepsilon) \sum_{j} \sum_{k=1}^{m}\left(\lambda_{k}\left(\phi_{k, j}^{n}\right)-\lambda_{k}\left(\phi_{k, j+1}^{n}\right)\right)\left|v_{j}^{n}\right|^{2}+O(1) \varepsilon^{3} e^{-O(1) \varepsilon n} \\
& +O(1) \sum_{j} \sum_{k=1}^{m}\left(\lambda_{k}\left(\phi_{k, j}^{n}\right)-\lambda_{k}\left(\phi_{k, j+1}^{n}\right)\right)\left|v_{j}^{n}\right|\left|v_{j+1}^{n}-v_{j}^{n}\right| \\
& +O(1) \sum_{j} \varepsilon^{2} e^{-O(1) \varepsilon(|j|+n)}\left|v_{j}^{n}\right|
\end{aligned}
$$

We handle the last term on the right-hand side of (5.18) in the same way as in the proof of Lemma 5.2 to obtain

$$
O(1) \sum_{j} \varepsilon^{2} e^{-O(1) \varepsilon(|j|+n)}\left|v_{j}^{n}\right| \leqq \varepsilon^{1 / 4} \sum_{j, n \leqq n_{2}}\left|v_{j}^{n}-v_{j+1}^{n}\right|^{2}+O(1) \varepsilon^{1 / 4} .
$$

Applying Cauchy's inequality gives

$$
\begin{aligned}
O(1) & \sum_{j} \sum_{k=1}^{m}\left(\lambda_{k}\left(\phi_{k, j}^{n}\right)-\lambda_{k}\left(\phi_{k, j+1}^{n}\right)\right)\left|v_{j}^{n}\right|\left|v_{j+1}^{n}-v_{j}^{n}\right| \\
\leqq & \frac{\lambda}{8} \sum_{j} \sum_{k=1}^{m}\left(\lambda_{k}\left(\phi_{k, j}^{n}\right)-\lambda_{k}\left(\phi_{k, j+1}^{n}\right)\right)\left|v_{j}^{n}\right|^{2}+O(\varepsilon) \sum_{j}\left|v_{j+1}^{n}-v_{j}^{n}\right| .
\end{aligned}
$$

Estimating (5.18), together with (5.19) and (5.20), yields (5.10). 
Proof of Lemma 5.4. First, as in the proof of Lemma 5.2, we have

$$
\lambda_{k}\left(\phi_{j}^{n}\right)=\lambda_{k}\left(\phi_{k, j}^{n}\right)+\sum_{\mu \neq k} \lambda_{k}\left(\phi_{\mu, j}^{n}\right)+O(1) \varepsilon^{2} e^{-O(1) \varepsilon(|j|+n)},
$$

so that the left-hand side of (5.11) becomes

$$
\begin{aligned}
& \sum_{k=1}^{m}\left(\lambda_{k}\left(\phi_{j}^{n}\right)-\lambda_{k}\left(\phi_{j+1}^{n}\right)\right)\left(v_{k, j}^{n}\right)^{2}=\sum_{k=1}^{m}\left(\lambda_{k}\left(\phi_{k, j}^{n}\right)-\lambda_{k}\left(\phi_{k, j+1}^{n}\right)\right)\left(v_{k, j}^{n}\right)^{2} \\
& \quad+\sum_{k=1}^{m} \sum_{\mu \neq k}\left(\lambda_{\mu}\left(\begin{array}{l}
n, j \\
k, j
\end{array}\right)-\lambda_{\mu}\left(\phi_{k, j+1}^{n}\right)\right)\left(v_{\mu, j}^{n}\right)^{2}+O(1) \varepsilon^{2} e^{-O(1) \varepsilon(|j|+n)}\left|v_{j}^{n}\right|^{2} \\
& \geqq \sum_{k=1}^{m}\left(\lambda_{k}\left(\phi_{k, j}^{n}\right)-\lambda_{k}\left(\phi_{k, j+1}^{n}\right)\right)\left(v_{k, j}^{n}\right)^{2} \\
& \quad-O(1) \sum_{k=1}^{m} \sum_{\mu \neq k}\left(\lambda_{k}\left(\phi_{k, j}^{n}\right)-\lambda_{k}\left(\phi_{k, j+1}^{n}\right)\right)\left(v_{\mu, j}^{n}\right)^{2} \\
& \quad-O(1) \varepsilon^{2} e^{-O(1) \varepsilon(|j|+n)}\left|v_{j}^{n}\right|^{2} .
\end{aligned}
$$

This, together with the estimate

$$
\begin{aligned}
\sum_{j, n \leqq n_{2}} O(1) \varepsilon^{2} e^{-O(1) \varepsilon(|j|+n)}\left|v_{j}^{n}\right|^{2} & \leqq \sum_{j, n \leqq n_{2}} O(1) \varepsilon^{2} e^{-O(1) \varepsilon(|j|+n)}\left|v_{j}^{n}\right| \\
& \leqq \varepsilon^{1 / 4} \sum_{j, n \leqq n_{2}}\left|v_{j}^{n}-v_{j+1}^{n}\right|^{2}+O(1) \varepsilon^{1 / 4}
\end{aligned}
$$

leads to $(5.11)$.

Proof of Lemma 5.5. We first estimate each element of the matrix on the lefthand side of (5.12). A Taylor expansion yields

$$
l_{k, \mu}\left(\phi_{j}^{n+1}\right)-l_{k, \mu}\left(\phi_{j}^{n}\right)=\nabla l_{k, \mu}\left(\phi_{j}^{n}\right)\left(\phi_{j}^{n+1}-\phi_{j}^{n}\right)+O(\varepsilon)\left|\phi_{j}^{n}-\phi_{j+1}^{n}\right| .
$$

Using Corollary 2.1 and (1.6), we have

$$
\phi_{j}^{n+1}-\phi_{j}^{n}=\sum_{\mu=1}^{m}\left(\phi_{\mu, j}^{n+1}-\phi_{\mu, j}^{n}\right)=\sum_{\mu=1}^{m} \frac{-\lambda s_{\mu}}{2}\left(\phi_{\mu, j+1}^{n}-\phi_{\mu, j-1}^{n}\right)+O(\varepsilon)\left|\phi_{j}^{n}-\phi_{j+1}^{n}\right| \text {. }
$$

Therefore,

$$
\begin{aligned}
& l_{k, \mu}\left(\phi_{j}^{n+1}\right)-l_{k, \mu}\left(\phi_{j}^{n}\right)+\frac{\lambda}{2} \lambda_{k}\left(\phi_{j}^{n}\right)\left(l_{k, \mu}\left(\phi_{j+1}^{n}\right)-l_{k, \mu}\left(\phi_{j-1}^{n}\right)\right) \\
& =O(1) \lambda \sum_{\mu=1}^{m}\left(\lambda_{k}\left(\phi_{j}^{n}\right)-s_{\mu}\right)\left(\phi_{\mu, j+1}^{n}-\phi_{\mu, j-1}^{n}\right)+O(\varepsilon)\left|\phi_{j}^{n}-\phi_{j+1}^{n}\right|=
\end{aligned}
$$




$$
\begin{aligned}
& =O(\varepsilon) \lambda\left|\phi_{k, j+1}^{n}-\phi_{k, j-1}^{n}\right|+O(1) \sum_{\mu \neq k}\left|\phi_{\mu, j+1}^{n}-\phi_{\mu, j-1}^{n}\right|+O(\varepsilon)\left|\phi_{j}^{n}-\phi_{j+1}^{n}\right| \\
& =O(\varepsilon) \lambda\left(\lambda_{k}\left(\phi_{k, j}^{n}\right)-\lambda\left(\phi_{k, j+1}^{n}\right)\right)+O(1) \sum_{\mu \neq k}\left(\lambda_{\mu}\left(\phi_{\mu, j}^{n}\right)-\lambda\left(\phi_{\mu, j+1}^{n}\right)\right) .
\end{aligned}
$$

Consequently,

$$
\begin{aligned}
& \sum_{j, n \leqq n_{2}} v_{j}^{n} \cdot\left(L_{j}^{n+1}-L_{j}^{n}+\frac{\lambda}{2} \Lambda_{j}^{n}\left(L_{j+1}^{n}-L_{j-1}^{n}\right)\right) R_{j}^{n} v_{j}^{n} \\
& \leqq O(\varepsilon) \lambda \sum_{j, n \leqq n_{2}} \sum_{k=1}^{m}\left(\lambda_{k}\left(\phi_{k, j}^{n}\right)-\lambda_{k}\left(\phi_{k, j+1}^{n}\right)\right)\left(v_{k, j}^{n}\right)^{2} \\
&+O(1) \lambda \sum_{j, n \leqq n_{2}} \sum_{k=1}^{m}\left(\lambda_{k}\left(\phi_{k, j}^{n}\right)-\lambda_{k}\left(\phi_{k, j+1}^{n}\right)\right)\left|v_{k, j}^{n}\right| \sum_{\mu \neq k}\left|v_{\mu, j}^{n}\right| \\
& \leqq \frac{\lambda}{8} \sum_{j, n \leqq n_{2}} \sum_{k=1}^{m}\left(\lambda_{k}\left(\phi_{k, j}^{n}\right)-\lambda_{k}\left(\phi_{k, j+1}^{n}\right)\right)\left(v_{k, j}^{n}\right)^{2} \\
&+O(1) \lambda \sum_{j, n \leqq n_{2}} \sum_{k=1}^{m}\left(\lambda_{k}\left(\phi_{k, j}^{n}\right)-\lambda_{k}\left(\begin{array}{c}
n \\
k, j+1
\end{array}\right)\right) \sum_{\mu \neq k}\left(v_{\mu, j}^{n}\right)^{2} .
\end{aligned}
$$

This is the desired estimate.

Finally we derive estimate (5.1) on the transversal waves. This can be done as in Proposition 3.1. For completeness, we sketch it here.

Proof of Proposition 5.1. Let $k$ be fixed, and let the $\mu$-th $(\mu \neq k)$ time-like grid line originating at $\left(j_{0}, 0\right)$ be defined by $(3.25)$ with $p$ replaced by $p_{\mu}$. In exactly the same way as in the proof of Proposition 3.1, we can show that

$$
\begin{aligned}
\sum_{j, n \leqq n_{2}}\left(\lambda_{k}\left(\phi_{k, j}^{n}\right)\right. & \left.-\lambda_{k}\left(\phi_{k, j+1}^{n}\right)\right) \sum_{\mu \neq k}\left(v_{\mu, j}^{n}\right)^{2} \\
\leqq & O(\varepsilon) \sum_{j}\left|v_{j}^{0}\right|^{2}+O(\varepsilon) \sum_{j, n \leqq n_{2}}\left|\phi_{j}^{n}-\phi_{j+1}^{n}\right|\left|v_{j}^{n}\right|^{2} \\
& +O(\varepsilon) \sum_{j, n \leqq n_{2}}\left|v_{j+1}^{n}-v_{j}^{n}\right|^{2}+O(1) \varepsilon^{4 / 3} .
\end{aligned}
$$

This inequality holds for each $k(1 \leqq k \leqq m)$. Summation over $k$ yields

$$
\begin{aligned}
& \sum_{j, n \leqq n_{2}} \sum_{k=1}^{m}\left(\lambda_{k}\left(\phi_{k, j}^{n}\right)-\lambda_{k}\left(\phi_{k, j+1}^{n}\right)\right) \sum_{\mu \neq k}\left(v_{\mu, j}^{n}\right)^{2} \\
& \leqq \\
& O(\varepsilon) \sum_{j}\left|v_{j}^{0}\right|^{2}+O(\varepsilon) \sum_{j, n \leqq n_{2}}\left|\phi_{j}^{n}-\phi_{j+1}^{n}\right|\left|v_{j}^{n}\right|^{2} \\
& \quad+O(\varepsilon) \sum_{j, n \leqq n_{2}}\left|v_{j+1}^{n}-v_{j}^{n}\right|^{2}+O(1) \varepsilon^{4 / 3}
\end{aligned}
$$


On the other hand, we have

$$
\begin{aligned}
\sum_{j, n \leqq n_{2}}\left|\phi_{j}^{n}-\phi_{j+1}^{n}\right|\left|v_{j}^{n}\right|^{2} \leqq & O(1) \sum_{j, n \leqq n_{2}} \sum_{k=1}^{m}\left(\lambda_{k}\left(\phi_{k, j}^{n}\right)-\lambda_{k}\left(\phi_{k, j+1}^{n}\right)\right)\left(v_{k, j}^{n}\right)^{2} \\
& +O(1) \sum_{j, n \leqq n_{2}} \sum_{k=1}^{m}\left(\lambda_{k}\left(\phi_{k, j}^{n}\right)-\lambda_{k}\left(\phi_{k, j+1}^{n}\right)\right) \sum_{\mu \neq k}\left(v_{\mu, j}^{n}\right)^{2} .
\end{aligned}
$$

It follows that

$$
\begin{aligned}
& \sum_{j, n \leqq n_{2}} \sum_{k=1}^{m}\left(\lambda_{k}\left(\phi_{k, j}^{n}\right)-\lambda_{k}\left(\phi_{k, j+1}^{n}\right)\right) \sum_{\mu \neq k}\left(v_{\mu, j}^{n}\right)^{2} \\
& \leqq O(\varepsilon) \sum_{j=-\infty}^{\infty}\left|v_{j}^{0}\right|^{2}+O(\varepsilon) \sum_{j, n \leqq n_{2}} \sum_{k=1}^{m}\left(\lambda_{k}\left(\phi_{k, j}^{n}\right)-\lambda_{k}\left(\phi_{k, j+1}^{n}\right)\right)\left(v_{k, j}^{n}\right)^{2} \\
&+O(\varepsilon) \sum_{j, n \leqq n_{2}}\left|v_{j+1}^{n}-v_{j}^{n}\right|^{2}+O(1) \varepsilon^{4 / 3}
\end{aligned}
$$

This completes the proof of Proposition 5.1.

Acknowledgments. We thank Professor PETER D. LAx for his interest and support in this work and Professor T.-P. LIU for helpful comments. J.-G. LIU's research was supported by DOE Grant DE-FG02-88ER-25053. XIN's research was supported in part by a Sloan Foundation Fellowship, NSF Grant DMS-90-02286, and DOE Grant DE-FG0288ER-25053.

\section{References}

1. I-LING CHERN, Large-time behavior of solutions of Lax-Friedrichs finite difference equations for hyperbolic systems of conservation laws, Math. Comp. 56 (1991), $107-118$.

2. M. G. CRANDALl \& A. MAJdA, Monotone difference approximations for scalar conservation laws, Math. Comp. 34 (1980), 1-21.

3. R. DiPerna, Convergence of approximate solutions to conservation laws, Arch. Rational Mech. Anal. 82 (1983), 27-70.

4. B. ENGquist \& S. OsHeR, One-sided difference approximations for nonlinear conservation laws, Math. Comp. 35 (1981), 321-351.

5. J. Goodman, Nonlinear asymptotic stability of viscous shock profiles to conservation laws, Arch. Rational Mech. Anal. 95 (1986), 325-344.

6. J. Goodman, Remarks on the stability of viscous shock waves, Viscous Profiles and Numerical Methods for Shock Waves, Edited by M. Shearer, SIAM, Philadelphia, 1991.

7. J. Goodman \& ZhoupIng XIN, Viscous limits for piecewise smooth solutions to systems of conservation laws, Arch. Rational Mech. Anal. 121 (1992), 235-265.

8. E. HARABETIAN, Rarefaction and large time behavior for parabolic equations and monotone schemes, Comm. Math. Phys. 114 (1988), 527-536.

9. G. H. Hardy, J. E. LitTlewood \& G. Polya, Inequalities, Cambridge University Press, London, New York, 1943. 
10. G. Jennings, Discrete shocks, Comm. Pure Appl. Math. 27 (1974), 25-37.

11. S. Kawashima \& A. Matsumura, Asymptotic stability of traveling wave solution to system for one dimensional gas motion, Comm. Math. Phys. 101 (1985), 97-127.

12. N. N. KuZnecov \& S. A. VološIn, On monotone difference approximations of a first order quasilinear equation, Soviet Math. Dokl. 17 (1976), 1203-1206.

13. P. D. LAX, Weak solutions of nonlinear hyperbolic equations and their numerical computation, Comm. Pure Appl. Math. 7 (1954), 159-193.

14. P. D. LAx, Hyperbolic Systems of Conservation Laws and the Mathematical Theory of Shock Waves, SIAM, Philadelphia, 1973.

15. TAr-Pina LiU, Nonlinear Stability of Shock Waves for Viscous Conservation Laws, Mem. Amer. Math. Soc. 328 Amer. Math. Soc., Providence, 1985.

16. JiAn-Guo LIU \& ZhouPING XIN, $L^{1}$-stability of stationary discrete shocks, Math. Comp. 60 (1993), 233-244.

17. Jian-Guo Liu \& Zhouping XIN, Convergence of Lax-Friedrichs scheme for piecewise smooth solutions to systems of conservation laws, in preparation.

18. A. MAJdA \& J. RALSTON, Discrete shock profiles for systems of conservation laws, Comm. Pure Appl. Math. 32 (1979), 445-483.

19. D. Michelson, Discrete shocks for difference approximations to systems of conservation laws, Adv. Appl. Math. 5 (1984), 433-469.

20. T. Nishida \& J. SMOlLER, A class of convergent finite-difference schemes for certain nonlinear parabolic systems, Comm. Pure Appl. Math. 36 (1983), 785-808.

21. O. OleINIK, Discontinuous solutions of nonlinear differential equations, Usp. Mat. Nauk. 12 (1957), 3-73.

22. S. OSHER \& J. RALSTON, $L^{1}$ stability of traveling waves with applications to convective porous media flow, Comm. Pure Appl. Math. 35 (1982), 737-751.

23. R. D. RichtmyER \& K. W. Morton, Difference Methods for Initial Value Problems, 2nd ed., Wiley-Interscience, New York, 1967.

24. R. SANDERS, On convergence of monotone finite difference schemes with variable spatial differencing, Math. Comp. 40 (1983), 91-106.

25. Y. S. SMYrLIS, Existence and stability of stationary profiles of the LW scheme, Comm. Pure Appl. Math. 42 (1990), 509-545.

26. A. SzEPESSY, On the stability of finite element methods for shock waves, Comm. Pure Appl. Math. 45 (1992), 923-946.

27. A. Szepessy \& ZhoupING XIN, Nonlinear stability of viscous shock waves, Arch. Rational Mech. Anal. 122 (1993), 53-103.

28. E. TADMOR, The large-time behavior of the scalar, genuinely nonlinear Lax-Friedrichs scheme, Math. Comp. 43 (1984), 353-368.

29. ZHoupING XIN, On the linearized stability of viscous shock profiles for systems of conservation laws, J. Diff. Eq. 100 (1992), 119-136.

Courant Institute of Mathematical Sciences

New York, New York 10012

(Accepted April 26, 1993) 